Pacific Journal of Mathematics

SURGERY WITH FINITE FUNDAMENTAL GROUP. I: TH 


\title{
SURGERY WITH FINITE FUNDAMENTAL GROUP I: THE OBSTRUCTIONS
}

\author{
R. JAMES MilgRAM
}

This paper determines the surgery obstructions for all surgery problems of the form

$$
\text { id } \times \sigma: M^{2} \times K^{4 k+2} \rightarrow M^{n} \times S^{4 k+2}
$$

as explicit elements in the surgery obstruction groups $L_{n+2}^{h}$ where $\sigma: K^{4 k+2} \rightarrow S^{4 k+2}$ is the usual Kervaire problem and $M^{n}$ is a closed, compact, oriented manifold with $\pi_{1}(M)$ finite. Due to the well known observation that the surgery obstruction for a surgery problem on a closed manifold depends only on the resulting cobordism class in $\Omega_{*}\left(B_{\pi_{1}(M)} \times G / T O P\right)$, this is the fundamental step in obtaining the surgery obstructions for all surgery problems over closed manifolds, as long as $\pi_{1}(M)$ is finite. (In the case $\pi_{1}(M)$ infinite, the situation is much more complex. A key part of the question would be resolved if one could prove the Novikov conjecture though.)

One of our main results is that only three types of obstruction can occur. This is, in fact, the first step in proving the oozing conjecture. The proof is completed in part II of this paper where we give characteristic class formulae for evaluating these obstructions.

In 1965 Dennis Sullivan proved a surgery product formula which made it possible to write down characteristic class formulae for evaluating the surgery obstructions of degree 1 normal maps over simply connected manifolds. Then in [W2] C. T. C. Wall made a preliminary study of the problem of characteristic class formulae for surgery in case the base is a closed manifold with finite fundamental group. He was able to show that formulae similar to the Sullivan result must exist in theory, and, moreover, are completely determined if they are known when the fundamental group is a finite 2-group.

W. Pardon [P] and G. Carlsson-R. J. Milgram [C-M] showed that if one looked at the surgery obstruction groups $L_{k}^{p}(\mathbf{Z} \pi)$ relevant to the problem obtained after crossing a given surgery problem with a circle $S^{1}$ then these groups are determined entirely by the rational representation ring of $\pi$ whenever $\pi$ is a finite 2-group. Based on this I. Hambleton $[\mathbf{H}]$, and independently B. Williams and Larry Taylor [T-W], gave an explicit identification of all possible obstructions for the $S^{1} \times($ ) problem, and showed that there were examples for which each was realized. However, the general case remained out of reach. 
In a sense this was surprising since Hambleton-Milgram [H-M], had already given effective methods using the $L^{p}($ ) groups above, together with a second explicit functor of the rational representation ring, to obtain descriptions of the surgery groups $L_{k}^{h}(\mathbf{Z}(\pi))$ relevant to the original problem. What resulted were approximately 23 distinct types of classes which could possibly be obstructions for problems over closed manifolds, and what was needed was a method for determining exactly which among them actually occur.

In this paper we consider this question in the basic case of products with the Kervaire problem, that is, we analyze all problems of the form

$$
\mathrm{id} \times \sigma: M^{n} \times K^{4 k+2} \longrightarrow M^{n} \times S^{4 k+2}
$$

where $\sigma: K^{4 k+2} \longrightarrow S^{4 k+2}$ is the simply connected Kervaire problem and $\pi_{1}\left(M^{n}\right)$ is finite. In these cases we give explicit recognition formulae for showing exactly which surgery obstructions are possible (exactly 3 of the types above).

In part II, [M4], of this paper we apply these results together with some explicit calculations to obtain cohomology characteristic class formulae in the manner of Sullivan's original theorem. As an immediate corollary we then have the proof of the (codimension 3 ) oozing conjecture (which will be explained further below).

In order to complete the study of surgery problems on closed manifolds with finite fundamental group there remains only the problem of studying the obstructions in $L_{k}^{h}\left(\mathbf{Z}\left(\pi_{1}\left(M^{n}\right)\right) ; \mathbf{Z} / 2\right)$ which arise from problems of the form

$$
\mathrm{id} \times \tau: N^{w} \times\left(S^{1} \times_{T} K^{4 k+2}\right) \longrightarrow N^{w} \times\left(S^{1} \times_{T} S^{4 k+2}\right)
$$

where $N^{w}$ is a $\mathbf{Z} / 2$-manifold and $\tau$ represents the twisted Kervaire problem [M2], [M-S], [T-W].

Implicit in our results on the ordinary Kervaire problem are the necessary techniques to analyze the twisted Kervaire problem. We do not discuss these results here primarily because the length of the paper would become excessive, but we will present them in part III [H-M-T-W], which represents joint work with Hambleton, Taylor, and Williams.

Among the consequences of these results are an effective determination of the set of homotopy triangulations of a given manifold $M$ which completes the classification theory for all oriented manifolds 
with finite fundamental group, since it gives an effective determination of the map $\partial$ in the surgery exact sequence

$$
\begin{aligned}
\ldots & \stackrel{\partial}{\rightarrow} L_{n+1}^{h}\left(\mathbf{Z} \pi_{1}\left(M^{n}\right)\right) \rightarrow \mathbf{H T}\left(M^{n}\right) \\
& \rightarrow\left[M^{n}, G /\{T O P \text { or } P L\}\right] \stackrel{\partial}{\rightarrow} L_{n}^{h}\left(\mathbf{Z}\left(\pi_{1}\left(M^{n}\right)\right) .\right.
\end{aligned}
$$

Additionally, using the Quinn-Ranicki assembly maps there are spec$\operatorname{tra} \mathbf{L}(\mathbf{Z}(\pi))$ whose homotopy groups are the surgery groups $L_{k}^{h}(\mathbf{Z}(\pi))$. The surgery exact sequence above can be interpreted in terms of a map of spectra

$$
\partial: M_{+} \wedge \mathbf{G} /(\mathbf{T O P} \text { or } \mathbf{P L}) \longrightarrow \mathbf{L}(\mathbf{Z}(\pi))
$$

and the cofiber of $\partial$ is a spectrum closely connected to $F(M) /$ Block Homeo $(M)$, where $F(M)$ is the space of homotopy equivalences of $M$ and Block Homeo $(M)$, the space of block homeomorphisms of $M$. In this respect, our results lead to an explicit determination of $\partial$ on the space level as well, and consequently to an explicit determination of the homotopy type of the cofiber. Again, we defer a complete exposition of these results to the sequels.

Before we describe the main result of part I we give more detail on the history of the problem.

It had become quite clear in the mid ' 70 's that the key question was to evaluate the obstruction for problems of the form 0.1. In view of Sullivan's Characteristic Variety Theorem the original way of thinking about these obstructions was to try to relate them to simply connected obstructions on various codimension $k$ submanifolds of $M^{n}$.

From this point of view J. Morgan originated the so called oozing conjecture which, in its original form, asserted that codimension 2 submanifolds would suffice. This was shown to be false by CappellShaneson [C-S], when they showed that the surgery problem

$$
\left(S^{3} / Q_{8}\right) \times K^{4 i+2} \longrightarrow\left(S^{3} / Q_{8}\right) \times S^{4 i+2}
$$

was non-trivial and had to be detected on a codimension 3 submanifold. (Here, $Q_{8}$ is the ordinary quaternion 8 group, and the action on $S^{3}$ is the usual one.) However, the only other types of examples known at that time were the original example of C.T.C. Wall [W3]

$$
\mathbf{R P}^{4 i+1} \times K^{4 k+2} \longrightarrow \mathbf{R P}^{4 i+1} \times S^{4 k+2}
$$

which he proved represented the non-trivial element in

$$
L_{3}^{h}(\mathbf{Z}(\mathbf{Z} / 2))=L_{3}^{p}(\mathbf{Z}(\mathbf{Z} / 2))=\mathbf{Z} / 2,
$$


and the somewhat unexpected example of Morgan-Pardon (see e.g. [M-R] for a discussion) which has the form

$$
\mathbf{R P}^{4 i+1} \times L_{4}^{4 j+1} \times K^{4 k+2} \longrightarrow \mathbf{R P}^{4 i+1} \times L_{4}^{4 j+1} \times S^{4 k+2}
$$

where $L_{4}^{4 j+1}$ is the $\mathbf{Z} / 4$-Lens space $S^{4 k+1} /(\mathbf{Z} / 4)$. This example represents the non-trivial torsion element in

$$
L_{0}^{h}(\mathbf{Z}(\mathbf{Z} / 2 \times \mathbf{Z} / 4))=\mathbf{Z}^{6} \oplus \mathbf{Z} / 2 .
$$

In [H], [T-W] the question was understood for $L_{k}^{p}(\mathbf{Z}(\pi))$. The result was that either the problem was already non-trivial when regarded as a simply-connected problem (hence determined by the previous results of Sullivan) or there was a homomorphism

$$
\phi: \pi \longrightarrow \mathbf{Z} / 2
$$

and the induced (pushed foreward) surgery problem was non-zero and determined by the formulae of [W2]. (In particular, both the CappellShaneson example and the Morgan-Pardon example become trivial when crossed with $S^{1}$.)

This prompted an energetic attack on the general problem. First, new examples were actively sought. Then extensive studies of the homology of finite two-groups were attempted to see if there were some limits on the types of classes due to homological restrictions. Both attacks failed. This is not surprising since we will prove that all examples are either of the types above or are induced up to larger groups from subquotients where they have the types above.

Assume that we are given a closed, oriented, manifold $M$ together with a homomorphism $f: \pi_{1}(M) \rightarrow \pi$ for a fixed group $\pi$. This data determines a surgery obstruction class $\sigma_{f}(\mathrm{id} \times \sigma) \in L_{\operatorname{dim}(M)+2}^{h}(\mathbf{Z} \pi)$. If $N$ is a subquotient of $\pi$, i.e. there is a subgroup $H \subset \pi$ and a surjection $\phi: H \rightarrow N$,

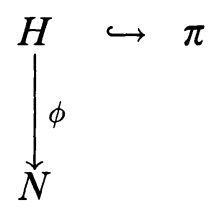

then there is a corresponding operation on $M:$ take the induced $H$ cover $\widetilde{M}$ with fundamental group $f^{-1}(H)$, together with the homomorphism $\phi \circ f \mid: \pi_{1}(\widetilde{M}) \rightarrow N$,

$$
(M, f) \mapsto(\widetilde{M}, \phi \circ f \mid) .
$$


This gives a new surgery obstruction $\sigma_{\phi \circ f \mid}(\mathrm{id} \times \sigma) \in L_{\operatorname{dim}(M)+2}^{h}(\mathbf{Z} N)$. In what follows $|\pi|<\infty$ and we are only interested in two special classes of subquotients:

(1) $N$ is a quaternion group of order $2^{n}$, or

(2) $N$ is an abelian quotient of the 2-Sylow subgroup $\operatorname{Syl}_{2}(\pi)$ itself. Our first main result is

THEOREM 0.4. Let $(M, f)$ be given as above with $\pi$ finite and suppose $\operatorname{dim}(M) \not \equiv 2(4)$. Then the surgery obstruction $\sigma_{f}(\mathrm{id} \times \sigma)$ is non-trivial if and only if one of the obstructions $\sigma_{\phi \circ f \mid}(\mathrm{id} \times \sigma)$ is non-zero as $N$ runs over quaternion type subquotients as above, $\mathrm{Z} / 2$ quotients of $\operatorname{Syl}_{2}(\pi)$, or $\operatorname{dim}(M) \equiv 0(4)$ and the index of $M$ is odd.

(This is a combination of Theorem 6.1 and Corollary 8.7 in the body of the text.)

In more detail, the quaternion subquotients correspond to the Cappell-Shaneson example and give non-trivial elements only for

$$
\operatorname{dim}(M) \equiv 3(4),
$$

(6.1 and 6.12). The $\mathbf{Z} / 2$ quotients of $\operatorname{Syl}_{2}(\pi)$ correspond to Wall's example and give elements only for

$$
\operatorname{dim}(M) \equiv 1(4) \text {. }
$$

Moreover, the images $\sigma_{f}(\mathrm{id} \times \sigma)$ generate a subgroup of $L_{3}^{h}(\mathbf{Z} \pi)$ isomorphic to $H_{1}\left(B_{\pi} ; \mathbf{Z} / 2\right),(6.13)$. The remarkable fact that

$$
\sigma_{f}(\text { id } \times \sigma) \equiv 0 \text { if } \operatorname{dim}(M) \equiv 0(4)
$$

and the index of $M$ is even is demonstrated in $\S 8$. Of course, if the index of $M$ is odd, then Sullivan, in his thesis, showed that the simply connected surgery obstruction, $\sigma_{e \circ f}(\mathrm{id} \times \sigma)$ is non-zero where $e \circ f$ is the projection of $\pi_{1}(M)$ onto the trivial group.

If the dimension of $M$ is $\equiv 2(4)$ the situation is somewhat different. In order to explain it we must describe the techniques actually used to prove 0.4 .

We introduce certain intermediate $L$-groups, $L_{*}^{h}(\mathbf{Z}(\lambda) \pi)$, in $\S 1$ and show that the product formulae discussed above factor through them. More exactly, it is known, [W2], that the surgery obstruction above depends only on the bordism class of $(M, f)$ in $\Omega_{*}\left(B_{\pi}\right)$ and we construct homomorphisms

$$
e_{\lambda}: \Omega_{*}\left(B_{\pi}\right) \rightarrow L_{*}^{h}(\mathbf{Z}(\lambda) \pi),
$$


(see e.g. (1.17)), and homomorphisms $\tau_{K}: L_{*}^{h}(\mathbf{Z}(\lambda) \pi) \rightarrow L_{*+2}^{h}(\mathbf{Z} \pi)$ so that the composite $\tau_{K} \circ e_{\lambda}[(M, f)]=\sigma_{f}(\mathrm{id} \times \sigma)$. Most of our effort here is devoted to calculating the groups $L_{*}^{h}\left(\mathbf{Z}\left(\zeta_{3}\right) \pi\right.$ ) (a special case of $\lambda$ ), and the map $\tau_{K}$.

Within these intermediate groups we show that only the classes corresponding to the examples above survive to generate candidates for non-trivial product obstructions when $\operatorname{dim}(M) \neq 2(4)$. This gives most of the results above. Explicit calculations for certain model groups are necessary to complete the proof. These are given in $\S 8$.

When $\operatorname{dim}(M) \equiv 2(4)$ we find, corresponding to the Morgan-Pardon example above, that further cases are necessary. We prove

THEOREM 0.5. Let $\pi$ be finite, then

$$
\operatorname{im}\left(e_{\zeta_{3}}: \Omega_{4 i+2}\left(B_{\pi}\right) \rightarrow L_{2}^{h}\left(\mathbf{Z}\left(\zeta_{3}\right) \pi\right)\right)
$$

is contained in the torsion subgroup and there is a "kernel" $V_{2} \subset$ $\operatorname{tor}\left(L_{2}^{h}\left(\mathbf{Z}\left(\zeta_{3}\right) \pi\right)\right)$, natural with respect to restriction and projection, so that if $e_{\zeta_{3}}(M, f) \in V_{2}$ then $\tau_{K} \circ e_{\zeta_{3}}(M, f)$ is zero in $L_{*}^{h}(\mathbf{Z} \pi)$. Moreover, the composite map

$$
\Omega_{4 i+2}\left(B_{\pi}\right) \stackrel{e_{\zeta_{3}}}{\longrightarrow} \operatorname{tor}\left(L_{2}^{h}\left(\mathbf{Z}\left(\zeta_{3}\right) \pi\right)\right) \longrightarrow \operatorname{tor}\left(L_{2}^{h}\left(\mathbf{Z}\left(\zeta_{3}\right) \pi\right)\right) / V_{2}
$$

is non-zero on $\{(M, f)\} \in \Omega_{4 i+2}\left(B_{\pi}\right)$ if and only if there is a subquotient

$$
\mathbf{Z} / 2 \times \mathbf{Z} / 2 \stackrel{\phi}{\leftarrow} H \hookrightarrow \pi
$$

so that $e_{\zeta_{3}}(\widetilde{M}, \phi \circ f \mid) \neq 0$ in $\operatorname{tor}\left(L_{2}^{h}\left(\mathbf{Z}\left(\zeta_{3}\right) \mathbf{Z} / 2 \times \mathbf{Z} / 2\right)\right) / V_{2}=\mathbf{Z} / 2$.

(This comes from 6.1, (7.3) and 7.9.) Using (7.3) and 7.11 we see that, given a subquotient $\mathbf{Z} / 2 \times \mathbf{Z} / 2 \stackrel{\phi}{\leftarrow} H \hookrightarrow \pi$, the map above will be non-zero whenever there is a homology class $\beta \in H_{2}\left(B_{\pi} ; \mathbf{Z}\right)$ so that, under the composite map

$$
\Sigma^{0} B_{\pi} \stackrel{\operatorname{tr}}{\longrightarrow} \Sigma^{0} B_{H} \stackrel{\Sigma^{0} B_{\phi}}{\longrightarrow} \Sigma^{0} B_{\mathbf{Z} / 2 \times \mathbf{Z} / 2}
$$

we have $\operatorname{tr}_{*} \circ\left(\Sigma^{0} B_{\phi}\right)_{*}(\beta) \neq 0$ in $H^{2}\left(B_{\mathbf{Z} / 2 \times \mathbf{Z} / 2} ; \mathbf{Z}\right)=\mathbf{Z} / 2$. (In [M4] we show the converse.)

Thus we identify the image of $e_{\zeta_{3}}$ in $L_{2}^{h}\left(\mathbf{Z}\left(\zeta_{3}\right) \pi\right)$, and the resulting surgery obstructions are then given as

$$
\tau_{K} \mid: \operatorname{im}\left(e_{\zeta_{3}}\right) \longrightarrow L_{0}^{h}(\mathbf{Z} \pi)
$$

We know from the Morgan-Pardon example that $\tau_{K}$ is non-trivial in some cases. However, we do not, at this time, know exactly when an 
element in $\operatorname{im}\left(e_{\zeta_{3}}\right)$ lies in $\operatorname{Ker}\left(\tau_{K}\right)$ for an arbitrary finite 2-group $\pi$. Hopefully 0.6 will clarify this statement.

REMARK 0.6. The dihedral groups $D_{2^{n}}$ for $n \geq 3$ give examples where the map $e_{\zeta_{3}}: \Omega_{2}\left(B_{D_{2^{n}}}\right) \rightarrow L_{2}^{h}\left(\mathbf{Z}\left(\zeta_{3}\right) D_{2^{n}}\right)$ is non-trivial. In particular, these dihedral groups give examples in dimensions congruent to $2 \bmod (4)$ where non-trivial restriction and projection are necessary to detect what is happening. Unfortunately, for these classes the image of $\tau_{K}$ is zero, so they do not give examples where the actual surgery obstruction is non-zero. It is currently unknown whether such examples exist.

The intermediate groups, $L_{*}^{h}(\mathbf{Z}(\lambda) \pi)$, play a crucial role in part II, ([M4]), as well. Indeed, we will actually prove a strengthened form of the oozing conjecture there which holds at the level of the intermediate groups themselves and pushes forward to the surgery groups to give the original conjecture-that codimension 3 ooze suffices when $\pi$ is finite-as a corollary. In other words, in part II we will study the map $e_{\lambda}$ and, based on the calculation of the groups $L_{*}^{h}(\mathbf{Z} \pi)$ here, will give characteristic class formulae to determine $e_{\lambda}$.

It seems that these intermediate groups provide a new and powerful invariant for closed manifolds. By way of further illustration, in part III, ([H-M-T-W]), we will construct a second type of surgery factorization, using the same kinds of intermediate surgery groups. This factorization enables us to analyze the $\mathbf{Z} / 2$-surgery obstructions in (0.2). But that essentially completes the analysis of ( $h$-cobordism) classification when $\pi_{1}$ is finite!

There still remains the more delicate problem of $s$-cobordism classification, but the current techniques seem to even have implications here. For these intermediate $L$-groups it seems to be possible to analyze the $L_{k}^{s}\left(\right.$ ) groups. (We have already analyzed the $L_{k}^{\prime}($ ) groups here and only certain $S K_{1}($ ) groups need to be understood to get to the $s$-cobordism category, but results of Oliver [O] imply that this is within range.) Thus we appear to be very near complete understanding of classification for manifolds with finite fundamental group.

This work evolved primarily during visits at The University of Edinburgh, Northwestern University, and McMaster University. In particular I would like to thank J. Davis, I. Hambleton, A. Ranicki, and B. Williams for their help and hospitality.

1. The Clauwens factorization and its refinements. We begin by describing a universal construction based on the following observation which I believe is due to Max Karoubi. 
Proposition 1.1. If $\mathbf{A}$ is a ring with involution-for which there is a central element $\alpha$ with $\alpha+\bar{\alpha}=1$ then the natural inclusion

$$
L_{*}(\mathbf{A},-) \longrightarrow L^{*}(\mathbf{A},-)
$$

is an isomorphism where $L_{*}(\mathbf{A})$ is the surgery obstruction group and $L^{*}(\mathbf{A})$ is the Mishchenko-Ranicki symmetric L-group.

Proposition 1.1 holds with any of the usual decorations, $s, h, p$, or ' . Let $\mathbf{A}$ be any ring with involution. Define a new involution on the polynomial ring $\mathbf{A}[s]$ by setting $\overline{\alpha s^{i}}=\bar{\alpha}(1-s)^{i}$ and define the map

$$
v: L^{*}(\mathbf{A}) \longrightarrow L_{*}(\mathbf{A}[s])
$$

as the composite

$$
\left.L^{*}(\mathbf{A}) \stackrel{1 \otimes\langle s\rangle}{\longrightarrow} L^{*}(\mathbf{A}) \otimes L_{*}(\mathbf{Z}[s]) \stackrel{r}{\longrightarrow} L_{*}(\mathbf{A})[s]\right)
$$

where $r$ is the Ranicki pairing and $1 \otimes\langle s\rangle$ sends the $n$-dimensional symmetric Poincaré complex $\left(C, \phi_{*}^{j}\right),\left\{\phi_{k}^{j}: C^{n-j} \rightarrow C_{j+k}\right.$, satisfy the conditions

$$
\partial \phi_{k}+(-1)^{j} \phi_{k} \delta+(-1)^{n+k-1}\left(\phi_{k-1}+(-1)^{(n-j)(j+k)+s} \overline{\phi_{k-1}}\right)=0,
$$

where $\left.\delta_{C^{n-\jmath}}=(-1)^{j} \bar{\partial}: C^{n-j} \rightarrow C^{n-j+1}\right\}$ to the complex $\left(C, \phi_{*}^{j}\right) \otimes$ $(\mathbf{Z}[s], s)$. (Again, see [R2], in particular p. 91, for more details on the definitions and properties of $\left(C, \phi_{*}^{j}\right)$.) Explicitly, the correspondence is given by sending $C_{*}$ to the tensor product with $\mathbf{Z}[s]$, and $\phi_{*}^{j}$ to $\phi_{*}^{j} \otimes s$,

$$
(C, \phi) \rightarrow\left(C \otimes_{\mathbf{Z}} \mathbf{Z}[s], \phi_{*} \otimes s\right) .
$$

Before we can exploit (1.2) we need to examine a somewhat more general construction. The modules and maps we are about to introduce are not difficult when dealing with free $A$-modules, but we need them for a wider class of $A$-modules in our applications.

Assumptions. $\mathbf{A}$ is a unitary ring with involution $\tau, \mathbf{S} \subset \mathbf{A}$ is a central unitary subring fixed under $\tau, \mathbf{N}$ is a finitely generated free S-module, $\mathbf{p}(s) \in \mathbf{S}[s]$ is a polynomial fixed under the extended involution on $\mathbf{S}[s]$, (i.e. $\mathbf{p}(s)=\mathbf{p}(1-s)$ ).

Let $\alpha \in \operatorname{Hom}_{\mathbf{S}}(\mathbf{N}, \mathbf{N})$ satisfy $\mathbf{p}(\alpha)=0$, then we see that

(1) if $\beta \in \operatorname{Hom}_{\mathbf{S}}\left(\mathbf{N}^{*}, \mathbf{N}^{*}\right)$ is $1-\bar{\alpha},\left\{\mathbf{N}^{*}=\operatorname{Hom}_{\mathbf{S}}(\mathbf{N}, \mathbf{S})\right\}$, then $\mathbf{p}(\beta)=0$. 
(2) $\alpha$ defines an (obvious) action of $\mathbf{S}[s]$ on $\mathbf{N}$ by $\left(\sum b_{i} s^{i}\right)(n)=$ $\left(\sum b_{i} \alpha^{i}\right)(n), b \in \mathbf{S}$, which factors through the quotient $\mathbf{S}_{p}=$ $\mathbf{S}[s] /(\mathbf{p}(s))$.

(3) Similarly, $\beta$ defines an action of $\mathbf{S}_{p}$ on $\mathbf{N}^{*}$, and we will assume these actions in the remainder of this section.

We use the notation $\mathbf{A}_{p}=A \otimes_{\mathbf{S}} \mathbf{S}_{p}$, and define functors

$$
\otimes_{\alpha}:{ }_{\mathbf{A}_{p}} \mathbf{C} \longrightarrow{ }_{\mathbf{A}} \mathbf{C}, \quad \otimes_{\beta}:{ }_{\mathbf{A}_{p}} \mathbf{C} \longrightarrow{ }_{\mathbf{A}} \mathbf{C}
$$

by $\otimes_{\alpha}(\mathbf{M})=\mathbf{M} \otimes_{\mathbf{S}_{p}} N$, and $\otimes_{\beta}(\mathbf{M})=\mathbf{M} \otimes_{\mathbf{S}_{p}} N^{*}$. (This makes sense since $\mathbf{S}_{p}$ is central in $\mathbf{A}_{p}$.) Also, define a map $e_{\mathbf{c}}^{\prime}: \mathbf{M}^{*} \times N^{*} \rightarrow$ $\operatorname{Map}(\mathbf{M} \times N, \mathbf{A})$ by the rule

$$
\left[e_{\mathbf{c}}^{\prime}(\phi \times \rho)\right](m \times n)=\rho\left(\phi(m) \otimes_{\mathbf{L}_{p}} n\right)=\phi(m) \rho(n) .
$$

Lemma 1.4. (a) $e_{\mathbf{c}}^{\prime}$ factors through a map $e_{\mathbf{c}}: \mathbf{M}^{*} \otimes_{\mathbf{S}_{p}} N^{*} \rightarrow$ $\left(\mathbf{M} \otimes_{\mathbf{S}_{p}} N\right)^{*}$.

(b) $e_{\mathbf{c}}$ is an isomorphism if $\mathbf{M}$ is a projective $\mathbf{A}_{p}$ module.

(c) Suppose $\lambda \in \operatorname{Aut}_{\mathbf{S}}\left(\mathbf{N}, \mathbf{N}^{*}\right)$ and $\beta=\lambda \alpha \lambda^{-1}$, then $\phi: \mathbf{M} \rightarrow \mathbf{M}^{*}$ given implies that

$$
(\phi \times \lambda): \mathbf{M} \times M \longrightarrow \mathbf{M}^{*} \times N^{*}
$$

factors through a well defined map (denoted $\phi \otimes \lambda$ by a mild abuse of notation since the two tensor products are over Different actions)

$$
\phi \otimes \lambda: \mathbf{M} \otimes_{\mathbf{S}_{p}} N \longrightarrow \mathbf{M}^{*} \otimes_{\mathbf{S}_{p}} N^{*}
$$

and $(\phi \otimes \lambda)^{*}=\phi^{*} \otimes \lambda^{*}$ when $\mathbf{M}$ is projective.

Proof. To check (a) one verifies the following three equations:

(1) $e_{\mathbf{c}}(\phi \times \rho)(s m \times n-m \times \alpha n)=0$

(2) $\left(e_{\mathbf{c}}(s \phi \times \rho)-e_{\mathbf{c}}(\phi \times \beta \rho)\right)(m \times n)=0$

(3) $\left(e_{\mathbf{c}}(a \phi \times \rho)\right)=a^{*}\left(e_{\mathbf{c}}(\phi \times \rho)\right)(m \times n)$.

To prove (b) it suffices to check on free modules since $\otimes$ preserves direct sums. Indeed, in this case the elements $e_{\mathrm{c}}(\phi \times \tau)$ are dense in the Hom group, and the finite dimensionality of $\mathbf{N}$ over $\mathbf{S}$ implies the result.

To prove (c) note that

$$
(\phi \times \lambda)(s m \times n-m \times \alpha n)=(s \phi(m) \times \lambda(n)-\phi(m) \times \beta \lambda(n))
$$

for all $m, n$ if and only if $\lambda \alpha=\beta \lambda$.

REMARK 1.5. $e_{\mathrm{c}}$ induces a natural transformation (again called $e_{\mathrm{c}}$ )

$$
e_{\mathbf{c}}: \otimes_{\alpha} \longrightarrow \otimes_{\beta}
$$

and on the subcategory of projectives $\left(e_{\mathrm{c}}\right)^{2}=$ id. 
EXAMPLE 1.6. Suppose an isomorphism $\lambda: \mathbf{N} \rightarrow \mathbf{N}^{*}$ is given as $\lambda=$ $\theta \pm \theta^{*}$ (so $\theta$ represents a non-singular \pm symmetric quadratic form on $\mathbf{N}$ ). Then if we set $\alpha=\lambda^{-1} \theta$ we have $\beta=1-\alpha^{*}=1 \mp \theta^{*} \lambda^{-1}$ but $\theta^{*}= \pm(\lambda-\theta)$ so

$$
\beta=\theta \lambda^{-1}=\lambda \alpha \lambda^{-1},
$$

and non-singular quadratic forms over $\mathbf{S}$ provide non-trivial examples of the functors $e_{\mathbf{c}}, \otimes_{\alpha}, \otimes_{\beta}$ as discussed above.

Given $\theta$ as above we can define a mapping $\tau_{\theta}$ on quadratic complexes $\left(C_{*}, \phi_{*}\right)$ over $\mathbf{A}_{p}$ where the $C_{i}$ are projective $\mathbf{A}_{p}$ modules by the correspondence

$$
\tau_{\theta}\left(\left(C_{*}, \phi_{*}\right)\right)=\left(\otimes_{\alpha}\left(C_{*}\right), 1 \otimes \lambda e_{\mathbf{c}}\left(\phi_{0}\right)\right)
$$

and the $\phi_{i}$ are maps

$$
C^{n-*-i} \longrightarrow C_{*},
$$

with $\phi_{0}$ a chain map. By naturality we thus have

THEOREM 1.7. $\tau_{\theta}$ induces maps of L-groups,

$$
\tau_{\theta}: L_{*}^{s, h, p}\left(\mathbf{A}_{p}\right) \longrightarrow L_{*+(1 \mp 1)}^{s, h, p}(\mathbf{A}) .
$$

Now, consider the composite

$$
\tau_{\theta} \mu: L^{*}(A) \longrightarrow L_{*+(1 \mp 1)}(A \otimes B) .
$$

THEOREM 1.9. The following diagram commutes

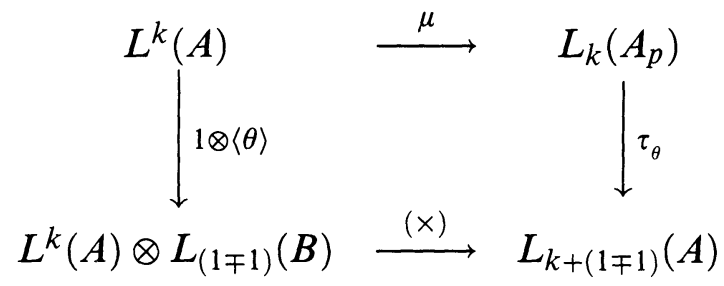

where $(\times)$ is the Ranicki product pairing.

Proof. If $(C, \phi)$ represents $\alpha \in L^{*}(A)$ then $\mu(\alpha)$ is represented by $\left(C \otimes_{A} A[s], \phi s\right)$. Consequently, $\tau_{\theta} \mu(C, \phi)$ is represented by $\left(\otimes_{\alpha}(C), e_{\mathbf{c}}(\phi s \otimes \lambda)\right)$, but

$$
e_{\mathbf{c}}(\phi s \otimes \lambda)=\left\{\phi_{0} \otimes \lambda \alpha\right\}=\left\{\phi_{0} \otimes \theta\right\},
$$

and the result follows since this is the definition of the Ranicki product $(x)$. 
REMARK 1.10 . In case $k$ is even and $\mathbf{p}=0$, the above construction and factorization of $(x)$ is a direct generalization of a construction due to F. B. J. Clauwens $[\mathbf{C l}]$. Of course, in that same paper Clauwens actually proves that his map $\tau_{\theta}$ depends only on the class of $\theta \in L_{(1 \mp 1)}(\mathbf{S})$ when $k$ is even. However, in the refined factorization above, $\tau_{\theta}$ most definitely does depend on the particular representative chosen within its class in $L_{(1 \mp 1)}(\mathbf{S})$, as we will see in Example 2.5.

In the remainder of this paper the key example is the Kervaire-Arf form

$$
K=\left(\begin{array}{ll}
1 & 1 \\
0 & 1
\end{array}\right)
$$

a quadratic reduction of the non-singular skew-form $\left(\begin{array}{cc}0 & 1 \\ -1 & 0\end{array}\right)=\lambda$. Note that

$$
\lambda^{-1} K=\left(\begin{array}{cc}
0 & -1 \\
1 & 0
\end{array}\right)\left(\begin{array}{ll}
1 & 1 \\
0 & 1
\end{array}\right)=\left(\begin{array}{cc}
0 & -1 \\
1 & 1
\end{array}\right)
$$

and this latter matrix satisfies the characteristic equation $\mathbf{p}(x)=x^{2}-$ $x+1$. But this is the irreducible equation of the 6 th root of unity $-\zeta_{3}$. Consequently

$$
\mathbf{Z}[s] /(\mathbf{p}(x))=\mathbf{Z}\left(\zeta_{3}\right),
$$

and from the naturality of the map $\tau_{K}$ in 1.9 we have

THEOREM 1.12. For the Kervaire form in (1.11) we have the factorization of the Ranicki product

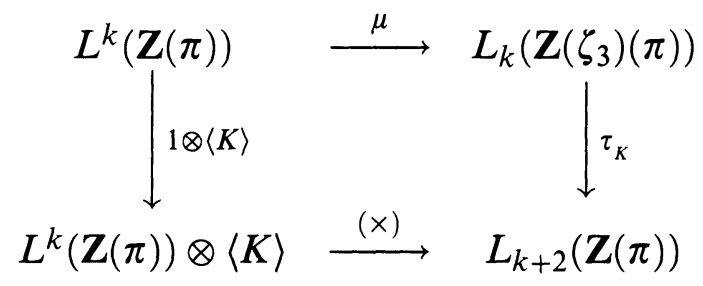

where $\langle K\rangle$ represents the Kervaire form and $\mathbf{Z}\left(\zeta_{3}\right) \pi$ is given the involution $\left(\sum \alpha_{i} g_{i}\right)^{*}=\sum \bar{a}_{i}\left(g_{i}\right)^{-1}$.

REMARK 1.13. There is nothing magical about $\mathbf{Z}\left(\zeta_{3}\right)$ in 1.12 . Indeed, if we choose alternate representatives for the Kervaire form we factor through other quadratic extensions of $\mathbf{Z}$. For example if we set

$$
\theta=\left(\begin{array}{ll}
1 & 1 \\
0 & n
\end{array}\right)
$$


then this also represents $\langle K\rangle$ when $n$ is odd, but the associated extension is

$$
\mathbf{Z}\left(\frac{1+\sqrt{(1-4 n)}}{2}\right) \text {, }
$$

and since $n$ is odd, $1-4 n$ is congruent to $5(\bmod 8)$. Thus, there will be a factorization for each quadratic extension

$$
\mathbf{Z}\left(\frac{1+\sqrt{v}}{2}\right)
$$

where $v \cong 5(\bmod 8)$. Indeed,

$$
\zeta_{3}=\frac{-1 \pm \sqrt{-3}}{2}
$$

is only singled out for convenience.

In [H-M-T-W] the factorization that will be used is through $\mathbf{Z}\left(\frac{1+\sqrt{5}}{2}\right)$, a ring in which the fundamental unit $\varepsilon=\frac{1+\sqrt{5}}{2}$ satisfies $\bar{\varepsilon}=-\varepsilon^{-1}$. But this implies that $L_{0}(\mathbf{Z}(\varepsilon),-)=\mathbf{Z} / 2$, and consequently $2 \times L_{*}(\mathbf{Z}(\varepsilon) \pi,-) \equiv 0$ for all $\pi$. This extra relation gives a factorization of the twisted Kervaire problem, and allows us to handle the remaining questions on surgery with finite fundamental group there. However, it turns out, [M-O], that the natural map $L_{*}^{h}(\mathbf{Z}[s] \pi,-) \rightarrow L_{*}^{h}\left(\mathbf{Z}\left(\zeta_{3}\right) \pi,-\right)$ is very close to being and injection, and hence, the information we obtain about $L_{*}^{h}\left(\mathbf{Z}\left(\zeta_{3}\right) \pi\right.$, - ) gives us the basic structure of the universal groups $L_{*}^{h}(\mathbf{Z}[s] \pi,-)$ themselves.

EXAMPLE 1.14. Let $C_{*}$ be the chain complex

$$
\mathbf{A}\left(\zeta_{3}\right)^{n}=C_{1} \stackrel{\partial}{\longrightarrow} C_{0}=\mathbf{A}\left(\zeta_{3}\right)^{n}
$$

for some $A\left(\zeta_{3}\right)$-map $\partial$, and $\left(C_{*}, \phi_{0}, \phi_{1}\right)$ be a non-singular symmetric complex representing the formation $\left(C_{1} \oplus C^{1}, C^{0}\right.$ ), (in Ranicki's terminology [R2], this means that the chain map $\phi_{0}$ is a chain homotopy equivalence, and the inclusion $C^{0} \rightarrow C_{1} \oplus C^{1}$ is just the pair $\left.\left(\phi_{0}^{1}, \delta\right)\right)$, then if we set

$$
\begin{gathered}
\phi_{i}=\phi_{1,1}+\phi_{1,2} \zeta_{3}, \\
\delta=\delta_{1}+\delta_{2} \zeta_{3},
\end{gathered}
$$

the representatives for $\tau_{K}\left(\left(C_{1} \oplus C^{1}, C^{0}\right)\right)$ are given by the maps

$$
\phi_{1,1}\left(\begin{array}{cc}
0 & 1 \\
-1 & 0
\end{array}\right)+\phi_{2,1}\left(\begin{array}{ll}
1 & 1 \\
0 & 1
\end{array}\right)
$$




$$
\delta_{1}\left(\begin{array}{ll}
1 & 0 \\
0 & 1
\end{array}\right)+\delta_{2}\left(\begin{array}{cc}
0 & -1 \\
1 & 1
\end{array}\right)
$$

Note in particular that the coboundary maps are what one would expect from just tensoring, but the matrices multiplying $\phi_{1,1}$ and $\phi_{2,1}$ have been modified by multiplying with $\lambda$.

REMARK 1.15. We should also note that the correspondence

$$
\otimes_{\lambda K}: \mathbf{M} \longrightarrow \mathbf{M} \otimes_{\lambda K} \mathbf{A}^{2}
$$

is just the forgetful map taking any $\mathbf{A}\left(\zeta_{3}\right)$-module $\mathbf{M}$ to the same $\mathbf{M}$ but now just regarded as an A-module.

The Ranicki pairings

$$
\begin{gathered}
L^{k}(\mathbf{A}) \otimes L^{s}(\mathbf{S}) \longrightarrow L^{k+s}(\mathbf{A}), \\
L^{k}(\mathbf{A}) \otimes L_{s}(\mathbf{S}) \longrightarrow L_{k+s}(\mathbf{A})
\end{gathered}
$$

behave nicely with respect to $\mu$. Indeed it follows directly from the definitions that the following diagram commutes

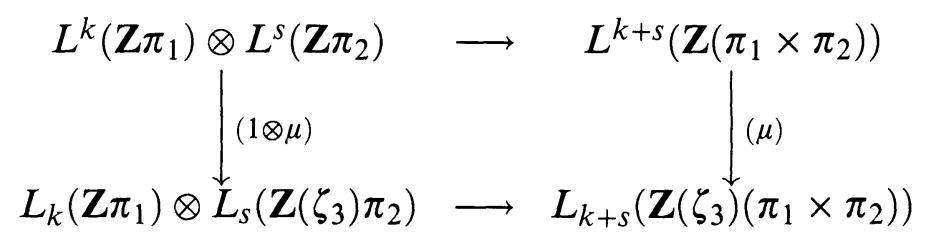

The case $\pi_{1}=1$ is especially important. Here, Ranicki's map from bordism to symmetric $L$-groups gives the comutative diagram

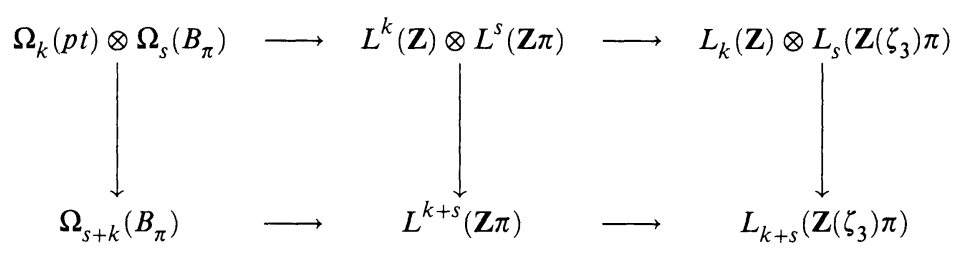

In particular, (1.17) plays a key role since for $k \geq 2$ Ranicki shows [R2] that

$$
L^{k}(\mathbf{Z})= \begin{cases}\mathbf{Z} & k=0(\bmod 4), \\ \mathbf{Z} / 2 & k=1(\bmod 4), \\ 0 & k=2(\bmod 4), \\ 0 & k=3(\bmod 4) .\end{cases}
$$

Note that the product pairing above implies that the class $\gamma$ in $L^{4 i+1}(\mathbf{Z})$ when tensored with the Kervaire problem $K$ must give 0 in $L_{4 i+3}(\mathbf{Z})=0$, so that, by the associativity of the product pairing, the product $\gamma b \otimes K=0$ in $L_{*}(\mathbf{Z} \pi)$ for any $b \in L^{*}(\mathbf{Z} \pi)$. Hence, we 
have

COROLLARY 1.18. $\tau_{K}(\gamma \beta)$ is not in the image of $L^{*}(\mathbf{Z} \pi) \otimes K$ for any $b \in L_{*}\left(\mathbf{Z}\left(\zeta_{3}\right) \pi\right)$.

REMARK 1.19. Recent work of W. Lück and A. Ranicki, [L-R], interprets the construction just given of $\tau_{K}$ as a transfer map associated to a specific quadratic form. They also construct transfers associated to quadratic formations $\left(H \oplus H^{*}, K\right)$, again see [R2] for a definition. These transfers have the form

$$
\tau_{\left(H \oplus H^{*}, K\right)}: L_{*}(A \pi) \longrightarrow L_{* \pm 1}(B \pi)
$$

for appropriate rings $A, B$. Indeed, this odd dimensional transfer is the actual tool used in [H-M-T-W] to study products with the twisted Kervaire problem. However, for that application, as was indicated in 1.13 , one can construct the required map explicitly, taking advantage of the fact that $\bar{\varepsilon}=-\varepsilon^{-1}$ gives an explicit reason for the triviality of multiplication by two in the group $L_{*}(\mathbf{Z}(\varepsilon) \pi,-)$. This was the approach originally taken by the author.

2. The evaluation of $\tau_{K}$. There is an involution defined on $K_{0}(A(\pi))$ by the rule $\{P\} \leftrightarrow\left\{P^{*}\right\}$, where, as usual, $P^{*}=\operatorname{Hom}_{A(\pi)}(P, A(\pi))$ made into a left $A(\pi)$ module via the involution on $A(\pi)$. This involution makes $K_{0}(A(\pi))$ into a $\mathbf{Z}(\mathbf{Z} / 2)$-module, and we have the Tate homology groups $\widehat{H}_{*}\left(\mathbf{Z} / 2 ; K_{0}(A(\pi))\right)$. Likewise, the involution gives rise to an involution on $K_{1}(A(\pi))$ and the quotient $\mathrm{Wh}(\mathbf{A}(\pi))=$ $K_{1}(\mathbf{A}(\pi)) /\{U(\mathbf{A}) \cdot \pi\}$ where $U(\mathbf{A})$ is the group of units in $\mathbf{A}$, induced from the operation of taking a matrix to its conjugate-transpose, and we have the Tate homology groups $\widehat{H}_{*}(\mathbf{Z} / 2 ; \mathrm{Wh}(\mathbf{A}(\pi))(A(\pi)))$. These groups are periodic of period 2 and become the relative terms in the exact sequences

$$
\begin{aligned}
\cdots & \longrightarrow \widehat{H}_{i}\left(\mathbf{Z} / 2 ; \widetilde{K}_{0}(A \pi)\right) \longrightarrow L_{i}^{h}(A \pi) \longrightarrow L_{i}^{p}(A \pi) \\
& \longrightarrow \widehat{H}_{i-1}\left(\mathbf{Z} / 2 ; \widetilde{K}_{0}(A \pi)\right) \longrightarrow L_{i-1}^{h}(A \pi) \longrightarrow \cdots \\
\cdots & \longrightarrow \widehat{H}_{i}(\mathbf{Z} / 2 ; \mathrm{Wh}(A \pi)) \longrightarrow L_{i}^{s}(A \pi) \longrightarrow L_{i}^{h}(A \pi) \\
& \longrightarrow \widehat{H}_{i-1}(\mathbf{Z} / 2 ; \mathrm{Wh}(A \pi)) \longrightarrow L_{i-1}^{s}(A \pi) \longrightarrow \cdots
\end{aligned}
$$

Let $A$ be one of the rings $\mathbf{Z}, \widehat{\mathbf{Z}}_{p}, \mathbf{Q}, \widehat{\mathbf{Q}}_{p}$, or a subring such aš $\mathbf{Z}(1 / 6)$. Let

$$
e: \widetilde{K}_{0}\left(A\left(\zeta_{3}\right) \pi\right) \longrightarrow \widetilde{K}_{0}(A \pi), \quad e: \mathrm{Wh}\left(A\left(\zeta_{3}\right) \pi\right) \longrightarrow \mathrm{Wh}(A \pi)
$$

be the forgetful maps. Specifically, regard an $A\left(\zeta_{3}\right)$-module $\mathbf{M}$ as an $A$-module, and $A\left(\zeta_{3}\right)$-module maps $\mathbf{M} \longrightarrow \mathbf{N}$ as $A$-maps. $e$ is 
involution preserving since inner automorphism induces the identity map on $K_{*}()$ and hence induces maps

$$
\begin{array}{ll}
e_{*}: & \widehat{H}_{*}\left(\mathbf{Z} / 2 ; \widetilde{K}_{0}\left(A\left(\zeta_{3}\right) \pi\right)\right) \longrightarrow \widehat{H}_{*}\left(\mathbf{Z} / 2 ; \widetilde{K}_{0}(A \pi)\right) \\
e_{*}: & \widehat{H}_{*}\left(\mathbf{Z} / 2 ; \mathrm{Wh}\left(A\left(\zeta_{3}\right) \pi\right)\right) \longrightarrow \widehat{H}_{*}(\mathbf{Z} / 2 ; \mathrm{Wh}(A \pi)) .
\end{array}
$$

We now have the preliminary

LEMMA 2.2. There are commutative diagrams

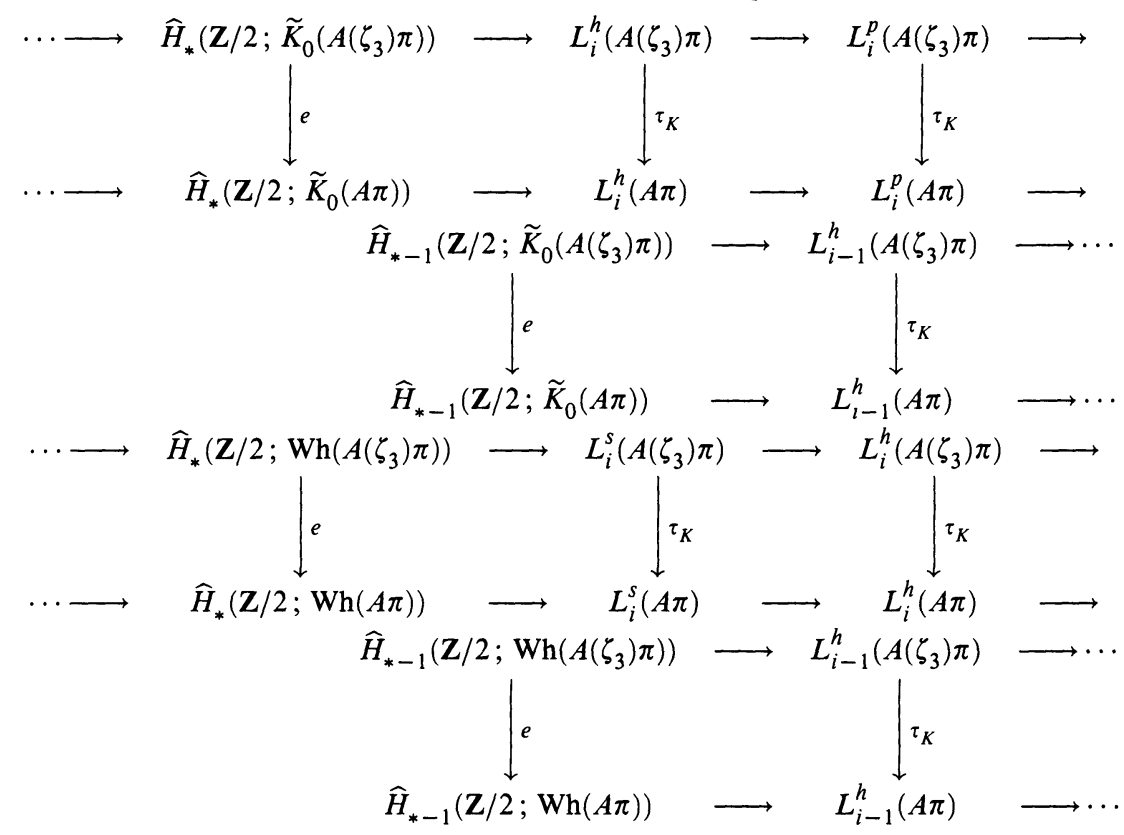

Proof. It suffices to check that the diagram commutes at the Tate homology groups. So first we recall the maps $\widehat{H}_{*}\left(\mathbf{Z} / 2 ; \widetilde{K}_{0}(B)\right) \rightarrow L_{*}^{h}(B)$. These are given as follows. If $*$ is even then $[P]$ represents $\beta$ in $\widehat{H}_{*}\left(\mathbf{Z} / 2 ; \widetilde{K}_{0}(B)\right)$ implies that $P \oplus P^{*}$ is free and we have that $\partial \beta=\left(P \oplus P^{*}, \phi\right)$ where $\phi(a, b)=b(a)$. Similarly, if $*$ is odd, then $[P]$ represents $\beta$ in $\widehat{H}_{*}\left(\mathbf{Z} / 2 ; \widetilde{K}_{0}(B)\right)$ means that $P=P^{*}$ and we have that

$$
\partial \beta=\left\{\left(P \oplus\left(-P^{*}\right)\right) \oplus\left(P^{*} \oplus(-P)\right), P \oplus-P\right\} .
$$

Suppose $*$ is even and $\beta \in L_{*}^{p}(B)$ is represented by the pair $(P, \phi)$ with $P$ a finitely generated projective and $\phi: P \longrightarrow P^{*}$ a $B$ map such that $\phi \pm \bar{\phi}=\lambda$ is an isomorphism. Then $\partial(P, \phi)=[P]$. On the other hand, if $*$ is odd and $\beta \in L_{*}^{p}(B)$ is represented by the formation $\left(P \oplus P^{*}, Q\right)$, then

$$
\partial\left(P \oplus P^{*}, Q\right)=[P]-[Q] .
$$


From these definitions it is clear that the diagrams above commute for the first sequence. The commutativity of the second sequence is similar.

Next we need

LEMMA 2.3. The diagram of localization sequences below commutes for $A=\mathbf{Z}$ or $\widehat{\mathbf{Z}}_{p}$ and $S$ any multiplicative set in $A$,

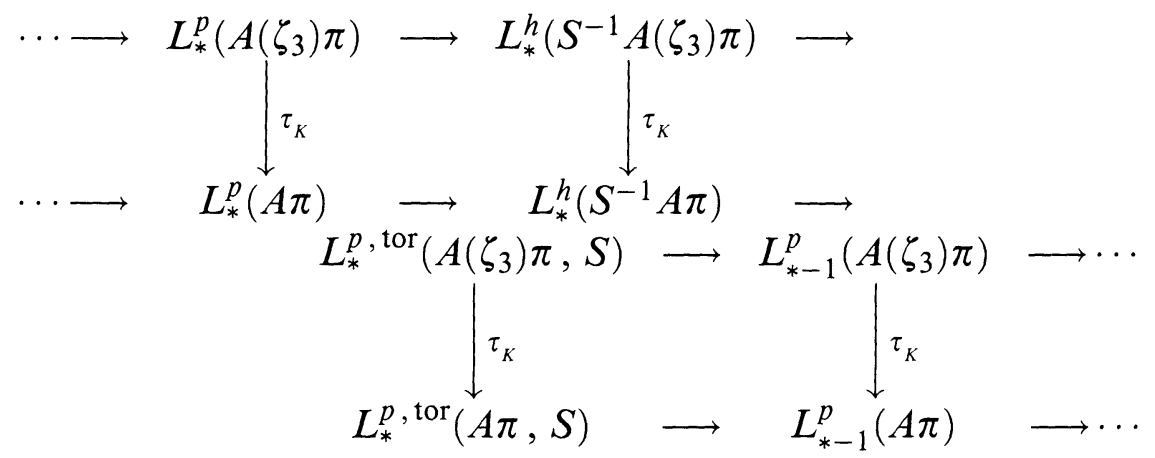

(This is direct from the fact that the definition of $\tau_{K}$ was carried out for chain complexes. See e.g. [R2] for the requisite naturality properties.)

Finally, we consider the case where $A$ is the field $\mathbf{Q}$ or $\widehat{\mathbf{Q}}_{p}$. In this case $L_{2 i}^{h}\left(A\left(\zeta_{3}\right) \pi\right)$ is the (Hermitian) Witt ring of the centers of the irreducible representations of $\pi$ while $L_{2 i+1}^{h}\left(A\left(\zeta_{3}\right) \pi\right)=0$, (see e.g. [C-M] for details), and the map

$$
\tau_{K}: L_{2 i}^{h}\left(A\left(\zeta_{3}\right) \pi\right) \longrightarrow L_{2 i+2}^{h}(A \pi)
$$

is given by first making the calculation in $L_{2 i}^{p}\left(A\left(\zeta_{3}\right) \pi\right)$ and then using the embedding of $L_{2 i}^{h}\left(A\left(\zeta_{3}\right) \pi\right)$ in $L_{2 i}^{p}\left(A\left(\zeta_{3}\right) \pi\right)$ to give the explicit final result. To do this recall that

$$
\zeta_{3} \longrightarrow-\left(\begin{array}{ll}
1 & 1 \\
0 & 1
\end{array}\right), \quad 1 \longrightarrow\left(\begin{array}{cc}
0 & 1 \\
-1 & 0
\end{array}\right)
$$

Then a form which diagonalizes to something of the type $\perp_{i}\left\langle a_{i} \zeta_{3}+b_{i}\right\rangle$ with the $a_{i}, b_{i}$ in the fixed field under the Galois automorphism $\left\{\zeta_{3} \leftrightarrow\left(\zeta_{3}\right)^{-1}\right\}$ will go to

$$
\perp_{i}\left\langle a_{i}\left(\begin{array}{cc}
-1 & -1 \\
0 & -1
\end{array}\right)+b_{i}\left(\begin{array}{cc}
0 & 1 \\
-1 & 0
\end{array}\right)\right\rangle \text {. }
$$

Of course, things are somewhat more complex in case the summand of $L_{2 i}^{p}\left(A\left(\zeta_{3}\right) \pi\right)$ in question is for example a matrix algebra over a 
non-trivial division algebra, but this is only because the $a_{i}$ and $b_{i}$ in the formula above will be elements in the division algebra in general, rather than in its center.

REMARK 2.5. Consider the class $w=\left\langle\zeta_{3}-\left(\zeta_{3}\right)^{-1}\right\rangle$ in $L_{2}\left(\mathbf{Q}\left(\zeta_{3}\right) \pi\right)$. We have

$$
\tau_{K}(w)=\left(\begin{array}{cc}
-1 & -1 \\
0 & -1
\end{array}\right)+\left(\begin{array}{cc}
-1 & 0 \\
-1 & -1
\end{array}\right)=\left(\begin{array}{cc}
-2 & -1 \\
-1 & -2
\end{array}\right) \sim\left(\begin{array}{cc}
-2 & 0 \\
0 & -3 / 2
\end{array}\right)
$$

and this class has index -2 , hence infinite order in $L_{0}(\mathbf{Q})$. In particular this shows that the Clauwens factorization mentioned in 1.10 cannot be independent of the representative of the Kervaire form for the extension to $L_{*}\left(\mathbf{Q}\left(\zeta_{3}\right) \pi\right)$. Moreover, since rationally $L_{*}\left(\mathbf{Z}\left(\zeta_{3}\right) \pi\right) \subset$ $L_{*}\left(\mathbf{Q}\left(\zeta_{3}\right) \pi\right)$, the map $\tau_{K}$ depends on the representative chosen for $K$ in $L_{*}\left(\mathbf{Z}\left(\zeta_{3}\right) \pi\right)$ as well!

3. Explicit calculations for $\pi$ a finite 2-group. We need some notation. Let $\zeta_{2^{i}}$ denote a primitive $2^{i}$ th root of unity, and set

$$
\lambda_{i}=\zeta_{2^{i}}+\zeta_{2^{i}}^{-1}, \quad \mu_{i}=\zeta_{2^{i}}-\zeta_{2^{i}}^{-1},
$$

then we have

Proposition 3.2. Let $\pi$ be a finite 2-group, then

$$
\mathbf{Q}\left(\zeta_{3}\right) \pi=\sum M_{n_{t}}\left(\mathbf{F}_{i}\right)
$$

where $\mathbf{F}_{i}$ is one of the three fields

$$
\mathbf{Q}\left(\zeta_{3}, \lambda_{i}\right), \quad \mathbf{Q}\left(\zeta_{3}, \mu_{i}\right), \quad \mathbf{Q}\left(\zeta_{3}, \zeta_{2^{l}}\right) .
$$

Proof. From $[\mathbf{F}]$ or $[\mathbf{M 1}]$ we have that

$$
\mathbf{Q}(\pi)=\sum M_{n_{i}}\left(\mathbf{L}_{i}\right) \oplus \sum M_{s_{i}}\left(\mathbf{D}_{i}\right)
$$

where $\mathbf{L}_{i}$ is one of the three fields $\mathbf{Q}\left(\lambda_{i}\right), \mathbf{Q}\left(\mu_{i}\right)$, or $\mathbf{Q}\left(\zeta_{2^{2}}\right)$, and $\mathbf{D}_{i}$ is the Quaternion algebra

$$
\mathbf{Q}\left(\lambda_{i}\right)\langle i, j\rangle /\left(i^{2}=j^{2}=-1, i j=-j i\right)=\mathbf{Q}\left(\lambda_{i}\right) \otimes \mathbf{D}_{1} .
$$

Hence, after tensoring with $\mathbf{Q}\left(\zeta_{3}\right)$,

$$
\mathbf{Q}\left(\zeta_{3}\right) \pi=\sum M_{n_{l}}\left(\mathbf{Q}\left(\zeta_{3}\right) \mathbf{L}_{i}\right) \oplus \sum M_{S_{l}}\left(\mathbf{Q}\left(\zeta_{3}\right) \otimes_{\mathbf{Q}} \mathbf{D}_{i}\right)
$$

We have

$$
\text { LeMma 3.3. } \mathbf{Q}\left(\zeta_{3}\right) \otimes_{\mathbf{Q}} \mathbf{D}_{i}=M_{2}\left(\mathbf{Q}\left(\zeta_{3}, \lambda_{i}\right)\right) \text {. }
$$

Proof. The Brauer invariants of $\mathbf{D}_{i}$ are $1 / 2$ at all the infinite places (and $1 / 2$ at 2 if there is only one infinite place). But at each infinite 
place tensoring with $\mathbf{Q}\left(\zeta_{3}\right)$ give a copy of $\mathbf{C}$, so the Brauer invariants there become zero. Likewise, $\widehat{\mathbf{Q}}_{2}\left(\lambda_{i}\right) \otimes \mathbf{Q}\left(\zeta_{3}\right)=\widehat{\mathbf{Q}}_{2}\left(\lambda_{i}, \zeta_{3}\right)$ is the degree 2 unramified extension of $\mathbf{Q}\left(\lambda_{i}\right)$, so at 2 as well the Brauer invariant becomes zero. Since all the Brauer invariants are now zero the result is a matrix algebra over its center, and 3.3 follows.

\section{3 implies 3.2 directly.}

We must be careful about the involution when we use the decomposition of 3.2. Every involution $\tau$ on $M_{n_{i}}\left(\mathbf{F}_{i}\right)$ which agrees with complex conjugation on the center $\mathbf{F}_{i}$ is equivalent to the usual involution (-) conjugate transpose, $M \rightarrow \bar{M}, m_{i j} \rightarrow \bar{m}_{j i}$. Precisely, there is a matrix $S$ so that

$$
S \bar{M} S^{-1}=\tau(M)
$$

Moreover, $\tau(S)= \pm S$, and we say that $\tau$ has type Ila if $S$ can be chosen so that $\tau(S)=S$. Otherwise, $\tau$ has type IIb.

LEMMA 3.4. $L_{i}\left(M_{n_{t}}\left(\mathbf{F}_{i}\right), \tau\right)=L_{i+2}\left(M_{n_{i}}\left(\mathbf{F}_{i}\right),{ }^{-}\right)$if $\tau$ has type IIb. Otherwise

$$
L_{i}\left(M_{n_{\imath}}\left(\mathbf{F}_{i}\right), \tau\right)=L_{i}\left(M_{n_{i}}\left(\mathbf{F}_{i}\right),-{ }^{-}\right.
$$

(See eg. [C-M] for a discussion.)

In the current case each simple summand of $\mathbf{Q} \pi$ is invariant under the involution so the same is true for each summand of $\mathbf{Q}\left(\zeta_{3}\right) \pi$. Moreover, each summand of $\mathbf{Q} \pi$ of the form $M_{n_{t}}\left(\mathbf{Q}\left(\lambda_{i}\right)\right), M_{n_{i}}\left(\mathbf{Q}\left(\mu_{i}\right)\right)$, or $M_{n_{i}}\left(\mathbf{Q}\left(\zeta_{2^{i}}\right)\right)$, on tensoring with $\mathbf{Q}\left(\zeta_{3}\right)$ gives rise to a IIa summand in $\mathbf{Q}\left(\zeta_{3}\right) \pi$ while each of the summands of type $M_{n_{i}}\left(\mathbf{D}_{i}\right)$ gives rise to a type IIb summand in $\mathbf{Q}\left(\zeta_{3}\right) \pi$. Thus, the distinction between the quaternion and matrix representations in $\mathbf{Q}\left(\zeta_{3}\right) \pi$ appears only in the shifting of their contributions to $L_{*}\left(\mathbf{Q}\left(\zeta_{3}\right) \pi\right)$ according to 3.4.

It should also be noted that 3.4 holds at all the completions of $\mathbf{F}_{i}$, and that $S$ above will be integral over $\mathbf{Z}\left(1 / 6, \zeta_{3}\right) \pi$. Consequently, we can use the Mayer-Vietoris sequence

$$
\begin{aligned}
\cdots & \rightarrow L_{k+1}^{h}\left(\widehat{\mathbf{Q}}_{2}\left(\zeta_{3}\right) \pi\right) \oplus L_{k+1}^{p}\left(\widehat{\mathbf{Q}}_{3}\left(\zeta_{3}\right) \pi\right) \rightarrow L_{k}^{p}\left(\mathbf{Z}\left(\zeta_{3}\right) \pi\right) \\
& \rightarrow L_{k}^{h}\left(\widehat{\mathbf{Z}}_{2}\left(\zeta_{3}\right) \pi\right) \oplus L_{k}^{p}\left(\widehat{\mathbf{Z}}_{3}\left(\zeta_{3}\right) \pi\right) \oplus L_{k}^{h}\left(\mathbf{Z}\left(1 / 6, \zeta_{3}\right) \pi\right) \\
& \rightarrow L_{k}^{h}\left(\widehat{\mathbf{Q}}_{2}\left(\zeta_{3}\right) \pi\right) \oplus L_{k}^{p}\left(\widehat{\mathbf{Q}}_{3}\left(\zeta_{3}\right) \pi\right) \rightarrow \cdots
\end{aligned}
$$

to calculate the groups $L_{k}^{p}\left(\mathbf{Z}\left(\zeta_{3}\right) \pi\right)$. 
We introduce the notation

$$
\begin{aligned}
& \lambda(\pi)=\text { number of } M_{n_{i}}\left(\mathbf{Q}\left(\lambda_{i}\right)\right) \text { summands of } \mathbf{Q} \pi, \\
& d(\pi)=\text { number of } M_{n_{i}}\left(\mathbf{D}_{i}\right) \text { summands of } \mathbf{Q} \pi, \\
& c(\pi)=\text { number of conjugacy classes in } \pi,
\end{aligned}
$$

and taking account of $3.2,3.4$ we obtain

THEOREM 3.7. The groups $L_{k}^{p}\left(\mathbf{Z}\left(\zeta_{3}\right) \pi\right)$ are given as follows,

$$
\begin{aligned}
& L_{1}^{p}\left(\mathbf{Z}\left(\zeta_{3}\right) \pi\right)=(\mathbf{Z} / 2)^{\lambda(\pi)-1} \\
& L_{3}^{p}\left(\mathbf{Z}\left(\zeta_{3}\right) \pi\right)=(\mathbf{Z} / 2)^{d(\pi)} \\
& L_{0}^{p}\left(\mathbf{Z}\left(\zeta_{3}\right) \pi\right)=L_{2}^{p}\left(\mathbf{Z}\left(\zeta_{3}\right)(\pi)\right)=\mathbf{Z}^{c(\pi)} .
\end{aligned}
$$

Proof. Let $A\left(\zeta_{3}\right) \pi$ be a direct sum $\amalg M_{n_{i}}\left(\mathscr{O}_{i}\left(\zeta_{3}\right)\right)$ with the involution either fixing each summand or interchanging them in pairs. (Here, as usual, $\mathscr{O}_{i}\left(\zeta_{3}\right)$ represents the ring of algebraic integers in the center of the $i$ th summand of $B\left(\zeta_{3}\right) \pi$ where $B$ is the quotient field of $A$.) Then Morita equivalence gives an isomorphism

$$
L_{k}^{p}\left(A\left(\zeta_{3}\right) \pi\right)=\coprod_{\substack{\text { fixed } \\ \text { summands }}} L_{k+2 \delta_{\imath}}^{p}\left(\mathscr{O}_{i}\left(\zeta_{3}\right) \pi\right)
$$

where $\delta_{i}=0$ or 1 as discussed above.

Moreover, whenever $1 / 3 \in A$, then multiplication by $\left\langle\zeta_{3}-\left(\zeta_{3}\right)^{-1}\right\rangle$ induces isomorphisms

$$
L_{k}^{p}\left(A\left(\zeta_{3}\right) \pi\right) \leftrightarrow L_{k+2}^{p}\left(A\left(\zeta_{3}\right) \pi\right)
$$

and $L_{2 k+1}^{p}\left(A\left(\zeta_{3}\right) \pi\right)=0$ whenever each $\mathscr{O}_{i}\left(\zeta_{3}\right)$ summand in (3.8) is a field $\mathbf{K}_{i}[\mathbf{R 1}]$. In this case note that the involution on $\mathbf{K}_{i}$ is a Galois automorphism and $\mathbf{F}_{i} \hookrightarrow \mathbf{K}_{i}$ is the fixed field.

We now use the prime decomposition of the fields $\mathbf{Q}\left(\zeta_{2^{\prime}}\right)$ to get the fields in the cases needed to apply (3.5).

For $i=2$ there is one prime over 3 , but for $i>2$ there are two primes interchanged by the involution. Hence there are also 2 primes over 3 in $\mathbf{Q}\left(\mu_{i}\right)$ interchanged by the involution, and there is only one prime over 3 in $\mathbf{Q}\left(\lambda_{i}\right)$. Finally, there is exactly one (totally ramified) prime over 2 for each of these fields.

When we adjoin $\zeta_{3}$ to any of these fields what happens at 3 is that the prime or primes there ramify with degree 2 , while at 2 we get the (unique) degree 2 non-ramified extension. 
Since there is only one prime over 2 in each of these fields, $L_{0}\left(\widehat{\mathbf{Q}}_{2} \otimes_{\mathbf{Q}} \mathbf{Q}_{2}\left(\zeta_{3}, v_{i}\right)\right)$ is identified with the Witt ring of Hermitian forms over $\widehat{\mathbf{Q}}_{2}\left(\zeta_{3}, v_{i}\right)$, so from [M-H] (Appendix 2) we have

$$
L_{0}\left(\widehat{\mathbf{Q}}\left(\zeta_{3}, v_{i}\right)\right)=\mathbf{Z} / 2 \oplus \mathbf{Z} / 2 \quad(i \geq 2)
$$

with generators $\langle 1\rangle$ and $\left\langle\pi_{i}\right\rangle$ where $\pi_{i}$ is either the uniformizing parameter for $\widehat{\mathbf{Q}}_{2}\left(\zeta_{3}, \zeta_{2^{\prime}}\right)$

$$
\pi_{i}=\left(\zeta_{3} \zeta_{2^{i}}+\left(\zeta_{3}\right)^{-1}\right)\left(\zeta_{2^{i}}^{-1}-1\right)
$$

or an appropriate norm of (3.9) if $v_{i}$ is $\lambda_{i}$ or $\mu_{i}$. Note for later reference that $\zeta_{3} \zeta_{2^{i}}+\left(\zeta_{3}\right)^{-1}$ is a global unit so the uniformizing parameter actually generates the prime ideal over 2 in $Z\left(\zeta_{3}, \zeta_{2^{\prime}}\right)$.

Since $\widehat{\mathbf{Q}}_{3}\left(\zeta_{3}\right)$ is a ramified extension of $\widehat{\mathbf{Q}}_{3}$, it follows that $\langle-1\rangle$ represents the non-norms so

$$
L_{0}\left(\widehat{\mathbf{Q}}_{3}\left(\zeta_{3}\right)\right)=\mathbf{Z} / 4 \text { with generator }\langle-1\rangle
$$

and, for $i>2$,

$$
L_{0}\left(\widehat{\mathbf{Q}}_{3}\left(\zeta_{3}, \lambda_{i}\right)\right)=\mathbf{Z} / 2 \oplus \mathbf{Z} / 2 \text {, generators }\langle 1\rangle,\left\langle m_{i}\right\rangle
$$

where $m_{i}$ represents a non-square in $F_{3^{(l)}}\left(l(i)=2^{i-2}\right)$. Since these are fields we have that the odd $L$-groups are identically zero [R1].

For the discrete valuation rings similar arguments (see e.g. [C-M]) give the table

\begin{tabular}{|l|l|l|}
\hline \multicolumn{1}{|c|}{$L$-group } & \multicolumn{1}{c|}{ Value } & Generator \\
\hline$L_{2 k+1}\left(\widehat{\mathbf{Z}}_{2}\left(\zeta_{3}\right) \pi\right)$ & 0 & \\
\hline$L_{2 k+1}\left(\widehat{\mathbf{Z}}_{3}\left(\zeta_{3}, v_{i}\right)\right)$ & 0 & \\
\hline$L_{2 k}\left(\widehat{\mathbf{Z}}_{2}\left(\zeta_{3}\right) \pi\right)$ & $\mathbf{Z} / 2$ & $\langle 1\rangle$ \\
\hline$L_{2}\left(\widehat{\mathbf{Z}}_{3}\left(\zeta_{3}, \lambda_{i}\right)\right)$ & 0 & \\
\hline$L_{0}\left(\widehat{\mathbf{Z}}_{3}\left(\zeta_{3}\right)\right)$ & $\mathbf{Z} / 4$ & $\langle-1\rangle$ \\
\hline$L_{0}\left(\widehat{\mathbf{Z}}_{3}\left(\zeta_{3}, \lambda_{l}\right)\right)(i \geq 3)$ & $\mathbf{Z} / 2 \oplus \mathbf{Z} / 2$ & $\langle 1\rangle,\left\langle m_{i}\right\rangle$ \\
\hline
\end{tabular}

Moreover, the last two cases in (3.10) map isomorphically to the corresponding groups for the quotient fields, while in the second case the $\mathbf{Z} / 2$ injects.

This reduces the effective use of (3.5) to the analysis of $L_{k}\left(\mathbf{Z}\left(1 / 6, \zeta_{3}\right) \pi\right)$ which we discuss now. To begin we need two results on the arithmetic of these rings.

LEMMA 3.11. The 2-primary part of $\widetilde{K}_{0}\left(\mathbf{Z}\left(\zeta_{3}, v_{i}\right)\right)$ is zero for $v_{i}=$ $\lambda_{i}, \mu_{i}$, or $\zeta_{2^{i}}$ (notation from (3.1)). 
Proof. It is well known that $\mathrm{Z}\left(\zeta_{3}\right)$ is a principal ideal domain, so its projective class group is trivial. But now, the extension

$$
\mathbf{Z}\left(\zeta_{3}, v_{i}\right) \hookleftarrow \mathbf{Z}\left(\zeta_{3}\right)
$$

is unramified at infinity and at the finite primes ramifies only at 2 which is totally ramified. Hence (3.12) satisfies the conditions of Theorem $10.4(\mathrm{a})$ of [WA], and as a result $\mid C L\left(\mathbf{Z}\left(\zeta_{3}, v_{i}\right) \mid\right.$ is prime to 2 as desired.

Also, recall that $\pi_{i}$ is the uniformizing parameter in the maximal real subfield of $\mathbf{Q}\left(\zeta_{3}, \zeta_{2^{\prime}}\right)$ given in (3.9). We have

LEMMA 3.13. (a) The units in the real subfield of $\mathbf{Q}\left(\zeta_{3}, \lambda_{i}\right) \bmod$ squares have independent signs at the various real places.

(b) The Galois submodule of $\mathbf{Q}\left(\zeta_{3}, v_{i}\right)^{\bullet}(\bmod$ squares $)$ generated by the uniformizing parameter $\pi_{i}$ or its norm (if $v_{i}=\mu_{i}$ ) consists of itself and all the units of the real subfield of $\mathbf{Q}\left(\zeta_{3}, v_{i}\right)$,

(c) The generators mod squares have independent signs at the various real places.

Proof. The first statement is clear since the real subfield is just $\mathbf{Q}\left(\lambda_{i}\right)$ and the assertion is well known (see e.g. [M3]). For the other cases consider the unit $\omega_{i}=\zeta_{3} \zeta_{2^{l}}-\left(\zeta_{3}\right)^{-1}$. Let $h \in \operatorname{Gal}\left(\mathbf{Q}\left(\zeta_{3 \times 2^{l}}\right) / \mathbf{Q}\right)$ be the Galois automorphism defined by $h\left(\zeta_{2^{l}}\right)=\zeta_{2^{l}}, h\left(\zeta_{3}\right)=\left(\zeta_{3}\right)^{2}$. Then $\omega_{i} h\left(\omega_{i}\right)=\zeta_{2^{i}}\left(1+\lambda_{i}\right)$. But this unit generates all the units of $\mathbf{Q}\left(\zeta_{2^{i}}\right)$ mod squares under the action of the Galois group, and it is also known that the real units in this set have independent signs at infinity. Thus, the map $u \rightarrow u h(u)$ takes the units mod squares in $\mathbf{Q}\left(\zeta_{3 \times 2^{i}}\right)$ onto the units mod squares in $\mathbf{Q}\left(\zeta_{2^{l}}\right)$. On the other hand

$$
\pi_{i} h\left(\pi_{i}\right)=\omega_{i} h\left(\omega_{i}\right)\left(\zeta_{2^{i}}+1\right)^{2}=\left(\zeta_{2^{l}}\right)^{-1}\left(\omega_{i} h\left(\omega_{i}\right)\right)\left(\zeta_{2^{i}}+1\right) \overline{\left(\zeta_{2^{i}}+1\right)},
$$

and since the last two terms contribute something totally positive the rest of the lemma follows.

These results together with the explicit calulations in $[\mathbf{M}-\mathbf{H}]$ for Hermitian forms, and the localization sequence with $S=$ all primes , gives

$$
\begin{gathered}
L_{\text {odd }}\left(\mathbf{Z}\left(1 / 6, \zeta_{3}, v_{i}\right)\right)=0 \\
L_{\mathrm{ev}}\left(\mathbf{Z}\left(1 / 6, \zeta_{3}, v_{i}\right)\right) \subset L_{\mathrm{ev}}\left(\mathbf{Q}\left(\zeta_{3}, v_{i}\right)\right)
\end{gathered}
$$

Thus

$$
L_{0}\left(\mathbf{Z}\left(1 / 6, \zeta_{3}, \lambda_{i}\right)\right)=\mathbf{Z} / 2 \oplus \mathbf{Z}^{d}
$$


where $d$ is the dimension of the fixed field $\mathbf{F}_{i}$. Generators are given by $\left\langle\pi_{i}\right\rangle-\langle 1\rangle$, and $\left\langle v_{i}\right\rangle$, where the $v_{i}$ run over the units of $\mathbf{F}_{i} \bmod$ squares. On the other hand when $v_{i}=\mu_{i}$ or $\zeta_{2^{i}}$ we have

$$
L_{0}\left(\mathbf{Z}\left(1 / 6, \zeta_{3}, v_{i}\right)=\mathbf{Z}^{d}\right.
$$

with generators given in 3.13 . Using these results, 3.7 is a direct application of (3.5).

REMARK 3.14. The classes in the groups $L_{\text {odd }}^{p}\left(Z\left(\zeta_{3}\right) \pi\right)$ come from the images of the classes $\left\langle\zeta_{3}-\zeta_{3}^{-1}\right\rangle$ in $L_{\mathrm{ev}}^{p}\left(\hat{\mathbf{Q}}_{3}\left(\zeta_{3}\right) \pi\right)$ at the appropriate representations in the exact sequence (3.5), and the $Z$ 's in $L_{\mathrm{ev}}^{p}\left(\mathbf{Z}\left(\zeta_{3}\right) \pi\right)$ are detected by the usual Hermitian multi-signatures on tensoring over $\mathbf{Q}\left(\zeta_{3}\right)$ with $\mathbf{C}$.

COROLlaRY 3.15. The groups $L_{k}^{h}\left(\mathbf{Z}\left(\zeta_{3}\right)\right)$ are

$$
\begin{cases}\mathbf{Z} & i=0, \\ 0 & i=1, \\ \mathbf{Z} & i=2, \\ 0 & i=3 .\end{cases}
$$

(This is direct from the proof of 3.7. The key calculation occurs at $*=2$, where

$$
L_{2}\left(\mathbf{Z}\left(1 / 6, \zeta_{3}\right)\right) \oplus L_{2}\left(\widehat{\mathbf{Z}}_{2}\left(\zeta_{3}\right)\right) \oplus L_{2}\left(\widehat{\mathbf{Z}}_{3}\left(\zeta_{3}\right)\right)=(\mathbf{Z} \oplus \mathbf{Z} / 2) \oplus(\mathbf{Z} / 2) \oplus(0)
$$

while

$$
L_{2}\left(\widehat{\mathbf{Q}}_{2}\left(\zeta_{3}\right)\right) \oplus L_{2}\left(\widehat{\mathbf{Q}}_{3}\left(\zeta_{3}\right)\right)=(\mathbf{Z} / 2 \oplus \mathbf{Z} / 2) \oplus(\mathbf{Z} / 4) .
$$

Just note that the image of $\langle\sqrt{-3}\rangle$ in $L_{2}\left(Z\left(1 / 6, \zeta_{3}\right)\right)$ is $\langle\sqrt{-3}\rangle_{2}+$ $\langle\sqrt{-3}\rangle_{3}$, and also, since $C L\left(\mathbf{Z}\left(1 / 6, \zeta_{3}\right)\right)=0$, it follows that $L^{h}()=$ $L^{p}()$.)

REMARK 3.16. We show in [M-O] that the groups

$$
L_{*}^{h}(\mathbf{Z}[s],-)=\left\{\begin{array}{ccl}
\mathbf{Z} & i=0 & \text { generator }\langle 1\rangle \\
\mathbf{Z} / 2 & i=1 & \text { generator } \gamma \\
0 & i=2 & \\
0 & i=3 &
\end{array}\right.
$$

and the reduction map gives an isomorphism in $L_{0}$.

4. The map $\tau_{K}: L_{k}^{p}\left(\mathbf{Z}\left(\zeta_{3}\right) \pi\right) \longrightarrow L_{k+2}^{p}(\mathbf{Z} \pi)$. A basic discovery, obtained more or less simultaneously by Carlsson-Milgram [C-M], Pardon $[\mathbf{P}]$, and (possibly) Kolster [K], was that when $\pi$ is a finite 2group, the groups $L_{k}^{p}(\mathrm{Z} \pi)$ depend only on the structure of the irreducible rational representations of $\pi$. A detailed summary of this 
dependence was given in $[\mathbf{H}-\mathbf{M}]$ as

THEOREM 4.1. Let $\pi$ be a finite 2-group and $\mathbf{Q} \pi=\sum_{\alpha} D_{\alpha}$ where the $D_{\alpha}$ are simple involuted algebras.

(1) There are groups $\Lambda_{i}\left(D_{\alpha}\right)$ depending only on the type of $D_{\alpha}$, the center of $D_{\alpha}$, and $i$ such that

$$
L_{i}^{p}(\mathbf{Z} \pi)=\sum_{\alpha} \Lambda_{i}\left(D_{\alpha}\right) \text { for } i=0,1,2 \text {, or } 3 .
$$

(2) Let $l(\alpha)$ be the number of simple summands in $D_{\alpha} \otimes_{\mathbf{Q}} \mathbf{R}$. The non-zero groups $\Lambda_{*}\left(D_{\alpha}\right)$ are:

(a) $\Lambda_{0}\left(D_{\alpha}\right)=(\mathbf{Z})^{l(\alpha)}$ for each $D_{\alpha}$.

(b) $\Lambda_{1}\left(D_{\alpha}\right)=(\mathbf{Z} / 2)^{2^{k-2}+1}$ if $D_{\alpha}$ has type SP and center $\mathbf{Q}\left(\lambda_{k}\right)$ for $k>1$.

(c) $\Lambda_{2}\left(D_{\alpha}\right)=(\mathbf{Z})^{l(\alpha)}$ if $D_{\alpha}$ has type $U ; \Lambda_{2}\left(D_{\alpha}\right)=\mathbf{Z} / 2$ if $D_{\alpha}=\mathbf{Q}$ with trivial G-action; $\Lambda_{2}\left(D_{\alpha}\right)=(\mathbf{Z} / 2)^{2^{k-2}-1}$ if $D_{\alpha}$ has type $S P$ and center $\mathbf{Q}\left(\lambda_{k}\right)$ for $k>2$.

(d) $\Lambda_{3}\left(D_{\alpha}\right)=\mathbf{Z} / 2$ if $D_{\alpha}$ has type $\mathbf{O}$ and $D_{\alpha} \neq \mathbf{Q}$ with trivial G-action.

REMARK 4.2. The $\mathbf{Z}$ 's in 4.1 are detected by signatures at the various real embeddings of the centers of the $D_{\alpha}$. The $\mathbf{Z} / 2$ 's in $L_{3}^{p}(\mathbf{Z} \pi)$ are represented in the Mayer-Vietoris sequence analogous to (3.5) by $\langle 3\rangle$ 's at the relevant representations, and the elements in $L_{1}^{p}(\mathbf{Z} \pi)$ are detected entirely by discriminants.

Using 4.1, 4.2 we now determine the map

$$
\tau_{K}: L_{k}^{p}\left(\mathbf{Z}\left(\zeta_{3}\right) \pi\right) \longrightarrow L_{k+2}^{p}(\mathbf{Z} \pi)
$$

The result is

Theorem 4.4. (a) The Z's in $L_{0}^{p}\left(\mathbf{Z}\left(\zeta_{3}\right) \pi\right)$ map to $\mathbf{Z}$ 's in $L_{2}^{p}(\mathbf{Z} \pi)$ except for the $\mathbf{Z}$ 's at the type IIa representations with center $\mathbf{Q}\left(\zeta_{3}, \lambda_{i}\right)$. The $\mathbf{Z}$ corresponding to the trivial representation maps onto the Kervaire-Arf class.

(b) The Z's in $L_{2}^{p}\left(\mathbf{Z}\left(\zeta_{3}\right) \pi\right)$ map into $L_{0}^{p}(\mathbf{Z} \pi)$ but none of them can be present in the image of bordism.

(c) The $\mathbf{Z} / 2$ 's in $L_{1}^{p}\left(\mathbf{Z}\left(\zeta_{3}\right) \pi\right)$ map to the non-trivial classes for the corresponding representations in $L_{3}^{p}(\mathbf{Z} \pi)$.

(d) The $\mathbf{Z} / 2$ 's in $L_{3}^{p}\left(\mathbf{Z}\left(\zeta_{3}\right) \pi\right)$ map to the classes with discriminant $\langle 3\rangle$ at the corresponding representations in $L_{1}^{p}(\mathbf{Z} \pi)$.

Proof. Note first that the maps

$$
L_{2 k}^{p}\left(\mathbf{Z}\left(\zeta_{3}\right) \pi\right) \longrightarrow L_{2 k}^{p}\left(\mathbf{Q}\left(\zeta_{3}\right) \pi\right), \quad L_{4}^{p}(\mathbf{Z} \pi) \longrightarrow L_{4}^{p}(\mathbf{Q} \pi)
$$


are injective, but

$$
\mathbf{Z} / 2 \longrightarrow L_{2}^{p}(\mathbf{Z} \pi) \longrightarrow L_{2}^{p}(\mathbf{Q} \pi)
$$

is an exact sequence where the $Z / 2$ kernel is represented by the simply connected Kervaire form (see e.g. [H-M] for details). Thus, except for the inverse image of the Kervaire form, the map (4.3) is completely determined by the rational map

$$
L_{2 k}^{p}\left(\mathbf{Q}\left(\zeta_{3}\right) \pi\right) \longrightarrow L_{2 k+2}^{p}(\mathbf{Q} \pi)
$$

but (4.5) is completely determined using formula (2.4). In particular for the type IIa summands of $\mathbf{Q}\left(\zeta_{3}\right) \pi$ the map is given on $L_{2}^{p}\left(\mathbf{Q}\left(\zeta_{3}\right) \pi\right)$ by the correspondence

$$
\left\langle u\left(\zeta_{3}-\left(\zeta_{3}\right)^{-1}\right)+v\right\rangle \longrightarrow\left\langle-u\left(\begin{array}{ll}
-2 & -1 \\
-1 & -2
\end{array}\right)+v\left(\begin{array}{cc}
0 & 1 \\
-1 & 0
\end{array}\right)\right\rangle .
$$

(4.6) simplifies markedly when made explicit at the various field summands of the center in $\mathbf{Q}\left(\zeta_{3}\right) \pi$. In particular note that the symmetry condition implies $u=0$ in $L_{0}\left(\mathbf{Q}\left(\zeta_{3}\right) \pi\right)$ while $v=0$ in $L_{2}\left(\mathbf{Q}\left(\zeta_{3}\right) \pi\right)$ whenever we are in a $M_{n_{i}}\left(\mathbf{Q}\left(\zeta_{3}, \lambda_{i}\right)\right)$ summand of type IIa. Thus we have

LEMMA 4.7. The map $\tau_{K}: L_{2 k}^{p}\left(\mathbf{Q}\left(\zeta_{3}, \lambda_{i}\right)\right) \longrightarrow L_{2 k+2}^{p}\left(\mathbf{Q}\left(\lambda_{i}\right)\right)$ is determined as follows:

(a) $\tau_{K}$ is 0 for $k$ even,

(b) $\tau_{K}\left(\left\langle a\left(\zeta_{3}-\left(\zeta_{3}\right)^{-1}\right)\right\rangle\right)=\langle-2 a\rangle+\langle-3 a / 2\rangle$ for $k$ odd.

When we are in an $M_{n_{i}}\left(\mathbf{Q}\left(\zeta_{3}, \mu_{i}\right)\right)$ or $M_{n_{i}}\left(\mathbf{Q}\left(\zeta_{3}, \zeta_{2^{2}}\right)\right)$ summand there exist elements such as $\mu_{i}$ which are themselves skew symmetric so that both $u, v$ can be non-zero in $L_{0}\left(\mathbf{Q}\left(\zeta_{3}\right) \pi\right)$ and $L_{2}\left(\mathbf{Q}\left(\zeta_{3}\right) \pi\right)$. For these cases we have

LEMMA 4.8. The map $\tau_{K}: L_{2 k}^{p}\left(\mathbf{F}\left(\zeta_{3}, \nu_{i}\right)\right) \rightarrow L_{2 k+2}^{p}\left(\mathbf{F}\left(\nu_{i}\right)\right)$ is given by the formula

$$
\left\langle u\left(\zeta_{3}-\left(\zeta_{3}\right)^{-1}\right)+v\right\rangle \longrightarrow\langle-2 u\rangle+\left\langle(1 /-2 u)\left(3 u^{2}+v^{2}\right)\right\rangle
$$

if $u$ is non-zero where $\nu_{i}$ is any one of $\lambda_{i}, \mu_{i}$ or $\zeta_{2^{\prime}}$ and $\mathbf{F}$ is any field containing $\mathbf{Q}$ but not containing $\zeta_{3}$. If $u=0$ then its image is 0 .

Proof. $\left\langle u\left(\zeta_{3}-\left(\zeta_{3}\right)^{-1}\right)+w\right\rangle \rightarrow\left(\begin{array}{cc}-2 u & w-u \\ -w-u & -2 u\end{array}\right)$. But this has determinant $3 u^{2}+w^{2}$ and hence diagonalizes to the form asserted in the statement of 4.7 if $u$ is non-zero. It is evidently hyperbolic when $u=0$. 
It remains to understand the case

$$
M_{2 n_{i}}\left(\mathbf{Q}\left(\zeta_{3}, \lambda_{i}\right)\right)=\mathbf{Q}\left(\zeta_{3}\right) \otimes M_{n_{i}}\left(D_{i}\right) .
$$

Using the Mayer-Vietoris sequence (3.5) and naturality which gives an evident set of commuting diagrams, this is a purely local problem for the fields $\widehat{\mathbf{Q}}_{2}$ and $\widehat{\mathbf{Q}}_{3}$. But unless we are at 2 and $\mathbf{D}_{i}$ is the ordinary quaternion algebra $\mathbf{D}_{1}$ where

$$
\mathbf{D}_{1}=\mathbf{Q}\langle i, j\rangle /\left(i^{2}=j^{2}=-1, i j=-j i\right)
$$

the $\mathbf{D}_{i}$ are just matrix algebras at these places, so the same formula as in 4.8 holds, though dimensions are reversed as was pointed out in 3.4 .

In the case of $\mathbf{D}_{1}$ the $\mathbf{Z} / 2$ in $L_{3}^{p}\left(\mathbf{Z}\left(\zeta_{3}\right) \pi\right)$ can be assumed to occur at the prime 3 , where $D_{1}$ is a matrix algebra. Hence, here too, 4.8 suffices to make the calculation.

To analyze the inverse image of the Arf invariant class in $L_{h}^{p}(\mathbf{Z} \pi)$ note that the composite

$$
\mathbf{A} \longrightarrow \mathbf{A}(\pi) \stackrel{\varepsilon}{\longrightarrow} \mathbf{A}
$$

is the identity, and this splits a natural direct summand $L_{*}^{p}(\mathbf{A})$ in $L_{*}^{p}(\mathbf{A} \pi)$. Of course, when $\mathbf{A}=\mathbf{Z}$, this splits off the Arf invariant class, and so it suffices to check things when $\pi=1$. But from the definitions in $1.6,1.7$, and $(1.11)$ of $\tau_{K}$, we have that $\tau_{K}(1)$ is the Kervaire class.

This completes the calculations for $k$ even.

In order to explain the calculations for $k$ odd we again use the Mayer-Vietoris sequence (3.5). It follows from 2.3 that the diagram

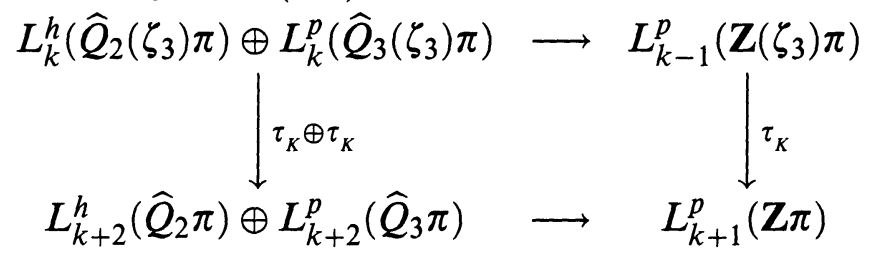

commutes. Moreover, in case $k$ is even (so $k-1$ is odd), the horizontal maps above are surjective. Thus, the odd case follows immediately from the even calculation. In particular, applying 4.8 completes the proof.

5. The structure of $\widetilde{K}_{0}\left(Z\left(\zeta_{3}\right) \pi\right)$. The $K$-theory localization sequence for $\widetilde{K}_{0}\left(\mathbf{Z}\left(\zeta_{3}\right) \pi\right)$ takes the form

$$
\begin{aligned}
\rightarrow K_{1}\left(\mathbf{Z}\left(\zeta_{3}\right) \pi\right) & \rightarrow K_{1}\left(\mathbf{Z}\left(1 / 2, \zeta_{3}\right) \pi\right) \oplus K_{1}\left(\widehat{\mathbf{Z}}_{2}\left(\zeta_{3}\right) \pi\right) \rightarrow K_{1}\left(\widehat{\mathbf{Q}}_{2}\left(\zeta_{3}\right) \pi\right) \\
& \stackrel{\partial}{\rightarrow} K_{0}\left(\mathbf{Z}\left(\zeta_{3}\right) \pi\right) \rightarrow K_{0}\left(\widehat{\mathbf{Z}}_{2}\left(\zeta_{3}\right) \pi\right) \oplus K_{0}\left(\mathbf{Z}\left(1 / 2, \zeta_{3}\right) \pi\right)
\end{aligned}
$$


and in the sequences $(2.1)$ what is required is $\widehat{H}_{*}\left(\mathbf{Z} / 2 ; \widetilde{K}_{0}\left(\mathbf{Z}\left(\zeta_{3}\right) \pi\right)\right)$. Hence only the 2-torsion in $\widetilde{K}_{0}\left(\mathbf{Z}\left(\zeta_{3}\right) \pi\right)$ is significant. It follows from 3.11 that the map $K_{1}\left(\widehat{\mathbf{Q}}_{2}\left(\zeta_{3}\right) \pi\right) \rightarrow \widetilde{K}_{0}\left(\mathbf{Z}\left(\zeta_{3}\right) \pi\right)$ in $(5.1)$ is onto. This reduces us to a basically local calculation, and we have

$$
\begin{aligned}
K_{1}\left(\widehat{Q}_{2}\left(\zeta_{3}\right) \pi\right) & =K_{1}\left(\coprod M_{n_{i}}\left(\widehat{Q}_{2}\left(\zeta_{3}, v_{i}\right)\right)\right) \\
& =\coprod K_{1}\left(\widehat{Q}_{2}\left(\zeta_{3}, v_{i}\right)\right)=\coprod \widehat{Q}_{2}\left(\zeta_{3}, v_{i}\right)^{\bullet}
\end{aligned}
$$

from 3.2 and the fact that there is only one prime over 2 in each of the fields $\mathbf{Q}\left(\zeta_{3}, v_{i}\right)$. For complete local fields the multiplicative structure of the non-zero elements is determined using the log and exponential map, and the following table results

\begin{tabular}{|c|l|}
\hline Field & \multicolumn{1}{c|}{ Units } \\
\hline$\widehat{Q}_{2}\left(\zeta_{3}, \lambda_{i}\right)$ & $\mathbf{Z} / 6 \times \mathbf{Z} \times \widehat{\mathbf{Z}}_{2}^{2^{i-2}}$ \\
\hline$\widehat{Q}_{2}\left(\zeta_{3}, \mu_{i}\right)$ & $\mathbf{Z} / 6 \times \mathbf{Z} \times \widehat{\mathbf{Z}}_{2}^{2^{i-2}}$ \\
\hline$\widehat{Q}_{2}\left(\zeta_{3}, \zeta_{2^{\prime}}\right)$ & $\mathbf{Z} /\left(3 \cdot 2^{i}\right) \times \mathbf{Z} \times \widehat{\mathbf{Z}}_{2}^{2^{l-1}}$ \\
\hline
\end{tabular}

In each case the $\mathbf{Z}$ is generated by the uniformizing parameter $\pi_{i}$ given in (3.9), or its norm to the appropriate subfield. The subgroup of $\widehat{Q}_{2}\left(\zeta_{3}, v_{i}\right)^{\bullet}$ of the form $\mathbf{Z} /\left(3 \cdot 2^{j}\right) \times \widehat{\mathbf{Z}}_{2}^{2^{k}}$ is called the group of units $U \widehat{Q}_{2}\left(\zeta_{3}, v_{i}\right)$.

Definition 5.2. The image of $K_{1}\left(\widehat{\mathbf{Z}}_{2}\left(\zeta_{3}\right) \pi\right)$ in $K_{1}\left(\widehat{\mathbf{Q}}_{2}\left(\zeta_{3}\right) \pi\right)$ is denoted $K_{1}^{\prime}\left(\widehat{\mathbf{Z}}_{2}\left(\zeta_{3}\right) \pi\right)$. The quotient $U K_{1}\left(\widehat{\mathbf{Q}}_{2}\left(\zeta_{3}\right) \pi\right) / K_{1}^{\prime}\left(\widehat{\mathbf{Z}}_{2}\left(\zeta_{3}\right) \pi\right)$ is written $K_{0}^{\prime}\left(\mathbf{Z}\left(\zeta_{3}\right) \pi\right)$.

We also need to keep track of the involution. The following result is well known.

LEMMA 5.3. The involution on $\widetilde{K}_{0}\left(\mathbf{Z}\left(\zeta_{3}\right) \pi\right),[P] \leftrightarrow\left[P^{*}\right]$, is the image of the involution on $K_{1}\left(\widehat{\mathbf{Q}}_{2}\left(\zeta_{3}\right) \pi\right)$ given by

$$
\left\{M_{i, j}\right\} \leftrightarrow\left\{\left(M_{j, i}^{*}\right)^{-1}\right\},
$$

where $M_{i, j}$ is a non-singular matrix with coefficients in $\widehat{\mathbf{Q}}_{2}\left(\zeta_{3}\right) \pi$.

The next result is our main technical tool, and is ultimately the reason we can understand the groups $L_{*}^{h}\left(\mathbf{Z}\left(\zeta_{3}\right) \pi,-\right)$ for $\pi$ a finite 2-group.

THEOREM 5.4. Let $\pi$ be a finite 2-group, then

$$
\widehat{H}_{*}\left(\mathbf{Z} / 2 ; K_{0}^{\prime}\left(\mathbf{Z}\left(\zeta_{3}\right) \pi\right)\right) \equiv 0 .
$$


Proof. The proof is in several steps. We first require

LEMMA 5.5. Let $\pi$ be a finite 2-group, then

$$
\widehat{H}_{*}\left(\mathbf{Z} / 2 ; U K_{1}\left(\widehat{\mathbf{Q}}_{2}\left(\zeta_{3}\right) \pi\right)\right) \equiv 0 .
$$

Proof (of 5.5). As was observed in 5.2

$$
K_{1}\left(\widehat{\mathbf{Q}}_{2}\left(\zeta_{3}\right) \pi\right)=\sum\left(\widehat{\mathbf{Q}}_{2}\left(\zeta_{3}, v_{i}\right)\right)^{\bullet},
$$

but this is even true with respect to the involution. Indeed, in each summand the involution acts as the usual Galois automorphism corresponding to complex conjugation $\left(^{-}\right)$followed by inversion $(\tau(\alpha)=$ $\left.\bar{\alpha}^{-1}\right)$. We denote the fixed field of $\left(^{-}\right)$by $\mathbf{F}_{i}$ and note that the extension $\hat{\mathbf{Q}}_{2}\left(\zeta_{3}, v_{i}\right) / \mathbf{F}_{i}$ is unramified. Hence, by Hilbert's theorem 90 it follows that $H_{\mathrm{ev}}\left(\mathbf{Z} / 2 ; K_{1}\left(\widehat{\mathbf{Q}}_{2}\left(\zeta_{3}\right) \pi\right)\right)=0$ where the action is now just conjugation. On the other hand, from local class-field theory,

$$
\mathbf{F}_{i}^{\bullet} / N\left(\widehat{\mathbf{Q}}_{2}\left(\zeta_{3}, v_{i}\right)^{\bullet}\right)=\mathbf{Z} / 2
$$

again where the action is only conjugation and, since the extension is unramified, the generator is the uniformizing parameter. Now since inversion only has the effect of shifting homology dimensions by 1 , and since, as we have seen

$$
\widehat{\mathbf{Q}}_{2}\left(\zeta_{3}, v_{i}\right)^{\bullet}=U \widehat{\mathbf{Q}}_{2}\left(\zeta_{3}, v_{i}\right) \times \mathbf{Z}
$$

where the generator of the $\mathbf{Z}$ is the uniformizing parameter $\pi_{i}$ which is fixed under conjugation from (3.9), the lemma follows.

Next we need to verify the result in the special case that $\pi$ is abelian. To do this we first require

LEMMA 5.6. Let $\pi$ be a finite abelian 2-group, then $K_{1}^{\prime}\left(\widehat{\mathbf{Z}}_{2}\left(\zeta_{3}\right) \pi\right)=$ $K_{1}\left(\widehat{\mathbf{Z}}_{2}\left(\zeta_{3}\right) \pi\right)$, and $H_{k}\left(\mathbf{Z} / 2 ; K_{1}\left(\widehat{\mathbf{Z}}_{2}\left(\zeta_{3}\right) \pi\right)\right) \equiv 0$ for all $k$.

Proof. Since $\pi$ is abelian the characterization of units $\varepsilon$ in $\widehat{\mathbf{Q}}_{2}\left(\zeta_{3}\right) \pi$ is that $e_{i} \varepsilon \neq 0$ for each central idempotent $e_{i}$. In particular, it follows that the units of $\widehat{\mathbf{Z}}_{2}\left(\zeta_{3}\right) \pi$ inject into the units of $\widehat{\mathbf{Q}}_{2}\left(\zeta_{3}\right) \pi$, and, since these are both local rings and the units generate $K_{1}()$, it follows that $K_{1}()=K_{1}^{\prime}()$. To prove the remainder of the lemma, note that $U\left(\widehat{\mathbf{Z}}_{2}\left(\zeta_{3}\right) \pi\right)=\mathbf{Z}_{3}(1+I)$ where $I$ is the augmentation ideal and the $Z_{3}$ represents the units in the coefficient ring which are not 
of the form $1+\theta$, with $\theta$ in the maximal ideal (2). In particular, the exponentiation operator

$$
\exp : 4 \widehat{\mathbf{Z}}_{2}\left(\zeta_{3}\right) \pi \longrightarrow U\left(\widehat{\mathbf{Z}}_{2}\left(\zeta_{3}\right) \pi\right)
$$

defined in the usual way by

$$
\exp (4 \theta)=\sum \frac{(4 \theta)^{i}}{n !}
$$

converges together with its reciprocal, the log operator, to provide an identification of $1+4 \widehat{\mathbf{Z}}_{2}\left(\zeta_{3}\right) \pi \subset K_{1}\left(\widehat{\mathbf{Z}}_{2}\left(\zeta_{3}\right) \pi\right)$ with $4 \widehat{\mathbf{Z}}_{2}\left(\zeta_{3}\right) \pi$ as abelian groups with involution. Moreover, since the involution on $4 \widehat{\mathbf{Z}}_{2}\left(\zeta_{3}\right) \pi$ is evidently free because it is already free on the coefficents $\widehat{\mathbf{Z}}_{2}\left(\zeta_{3}\right)$, we have reduced the lemma to showing that

$$
J_{4}=(1+I) /\left(1+4 \widehat{Z}_{2}\left(\zeta_{3}\right) \pi\right)
$$

has trivial homology under the involution.

There is an exact sequence

$$
0 \longrightarrow\left(2 \mathbf{Z}\left(\zeta_{3}\right) \pi / 4 \mathbf{Z}\left(\zeta_{3}\right) \pi\right)^{+} \longrightarrow \mathbf{Z} / 3 \times J_{4} \longrightarrow K_{1}\left(\mathbf{F}_{2}\left(\zeta_{3}\right) \pi\right) \longrightarrow 0
$$

and since $\left(2 \mathrm{Z}\left(\zeta_{3}\right) \pi / 4 \mathrm{Z}\left(\zeta_{3}\right) \pi\right)^{+} \cong \mathbf{F}_{2}\left(\zeta_{3}\right) \pi$ which has trivial Tate homology groups, it suffices to check that

$$
\widehat{H}_{*}\left(\mathbf{Z} / 2 ; K_{1}\left(\mathbf{F}_{2}\left(\zeta_{3}\right) \pi\right)\right) \equiv 0 \text {. }
$$

Let $I_{2} \subset \mathbf{F}_{2}\left(\zeta_{3}\right) \pi$ be the augmentation ideal. Then $I_{2}$ is nilpotent and there is a natural filtration $F^{i}\left(K_{1}\left(\mathbf{F}_{2}\left(\zeta_{3}\right) \pi\right)\right)$ defined by setting $F^{i}=$ image $\left(1+I_{2}^{i}\right)$. Then $F^{i} / F^{i+1} \cong I_{2}^{i} / I_{2}^{i+1}$. But $I_{2}^{i} / I_{2}^{i+1}$ is an $\mathbf{F}_{2}\left(\zeta_{3}\right)$ vector space. Indeed,

$$
I_{2}^{i} / I_{2}^{i+1} \cong\left(I\left(\mathbf{F}_{2} \pi\right)^{i} /\left(I \mathbf{F}_{2} \pi\right)^{i+1}\right) \otimes \mathbf{F}_{2}\left(\zeta_{3}\right)
$$

and from this the fact that $I_{2}^{i} / I_{2}^{i+1}$ has trivial homology is direct. Thus,

$$
H_{*}\left(\mathbf{Z} / 2 ; K_{1}\left(\mathbf{F}_{2}\left(\zeta_{3}\right) \pi\right)\right) \cong H^{*}\left(\mathbf{Z} / 2 ; K_{1}\left(\mathbf{F}_{2}\left(\zeta_{3}\right)\right)\right)=0
$$

and 5.6 follows.

COROLlary 5.7. Let $\pi$ be a finite 2 group, then

$$
\widehat{H}_{*}\left(\mathbf{Z} / 2 ; K_{1}^{\prime}\left(\widehat{\mathbf{Z}}_{2}\left(\zeta_{3}\right) \pi\right)\right) \equiv 0
$$


Proof. In [O] Oliver calculated the reduced Whitehead group of $\pi$ with coefficents in $\widehat{\mathbf{Z}}_{2}\left(\zeta_{3}\right) \pi$ and the result was described via a natural exact sequence

$$
0 \longrightarrow \mathrm{Wh}^{\prime}\left(\widehat{\mathbf{Z}}_{2}\left(\zeta_{3}\right) \pi\right) \stackrel{\Gamma}{\longrightarrow} \overline{\widehat{\mathbf{Z}}_{2}\left(\zeta_{3}\right) \pi} \longrightarrow \pi^{\mathrm{ab}} \longrightarrow 0
$$

where the middle term has trivial homology. If we consider the commutative diagram

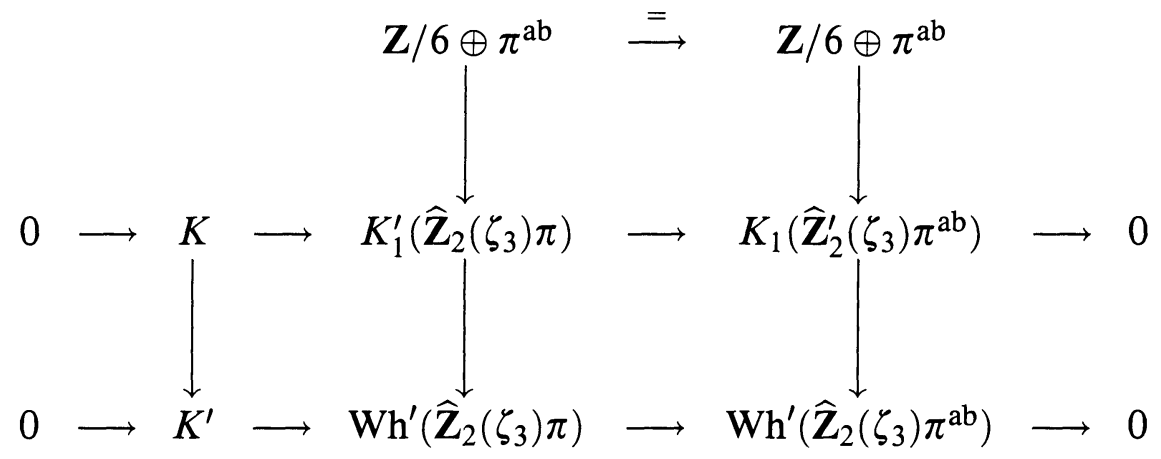

we see that $K$ and $K^{\prime}$ are isomorphic, where, of course, $K, K^{\prime}$ are the respective kernels. The naturality of Oliver's sequence implies that the homology of $K^{\prime}$ is trivial, hence the same is true of the homology of $K$, and using 5.6 the corollary follows.

But 5.7 and 5.5 immediately imply 5.4 and the proof is complete.

REMARK 5.8. There is an exact sequence

$$
\left.U K_{1}^{\prime}\left(\mathbf{Z}\left(1 / 2, \zeta_{3}\right) \pi\right)\right) \longrightarrow K_{0}^{\prime}\left(\mathbf{Z}\left(\zeta_{3}\right) \pi\right) \longrightarrow \widetilde{K}_{0}\left(\mathbf{Z}\left(\zeta_{3}\right) \pi\right)_{(2)} \longrightarrow 0
$$

which forms the heart of our analysis of the Tate homology groups $\widehat{H}_{*}\left(\mathbf{Z} / 2 ; \widetilde{K}_{0}\left(\mathbf{Z}\left(\zeta_{3}\right) \pi\right)\right)$, and 5.4 implies that these groups can be identified with the homology of the image of $U K_{1}\left(\mathbf{Z}\left(1 / 6, \zeta_{3}\right) \pi\right)$. Specifically we have

COROLlary 5.9.

$$
\widehat{H}_{*}\left(\mathbf{Z} / 2 ; \widetilde{K}_{0}\left(\mathbf{Z}\left(\zeta_{3}\right) \pi\right)\right) \cong \widehat{H}_{*+1}\left(\mathbf{Z} / 2 ; U^{\prime} K_{1}^{\prime}\left(\mathbf{Z}\left(1 / 2, \zeta_{3}\right) \pi\right)\right)
$$

where $U^{\prime} K_{1}^{\prime}\left(\mathbf{Z}\left(1 / 2, \zeta_{3}\right) \pi\right)$ is the image in $K_{0}^{\prime}\left(\mathbf{Z}\left(\zeta_{3}\right) \pi\right)$ of $U K_{1}^{\prime}\left(\mathbf{Z}\left(1 / 2, \zeta_{3}\right) \pi\right)$.

We now study $U K_{1}^{\prime}\left(\mathbf{Z}\left(1 / 2, \zeta_{3}\right) \pi\right)$. In Theorem 3.7 and its proof the units in $\mathbf{Z}\left(\zeta_{3}, v_{i}\right)$ mod squares were analyzed, but not as a module over conjugation. To understand this conjugation structure, note that the special unit $\varepsilon_{i}=\zeta_{3} \zeta_{2^{i}}^{-1}+\zeta_{3}^{-1}$ satisfies $\bar{\varepsilon}=\zeta_{2^{\prime}} \varepsilon$. Also, if $g\left(\zeta_{2^{i}}\right)=$ $-\left(\zeta_{2^{l}}\right)^{-1}$ so $\mathbf{Q}\left(\zeta_{2^{\prime}}\right)^{g}=\mathbf{Q}\left(\mu_{i}\right)$, then $(\varepsilon \times g(\varepsilon))^{*}=-\varepsilon \times g(\varepsilon)$. As an 
easy corollary we have

LEMMA 5.10. $U K_{1}^{\prime}\left(\mathbf{Z}\left(1 / 2, \zeta_{3}\right) \pi\right)$ is a direct sum of units at each irreducible $\mathbf{Q}\left(\zeta_{3}\right)$ representation of $\pi$. These units (as a module over conjugation followed by inversion) are given as follows:

(a) If the center is $\mathbf{Q}\left(\zeta_{3}, \zeta_{2^{l}}\right)$ then the module is $\left(\mathbf{Z}^{2^{l-1}-2}\right)^{-} \oplus$ $\left(\mathbf{Z} / 2^{i} \oplus \mathbf{Z}\right)$ where the last summand has generators $A, B$ with $2^{i} A=0$ and $\tau(A)=A, \tau(B)=A-B$.

(b) If the center is $\mathbf{Q}\left(\zeta_{3}, \mu_{i}\right)$ then the module is $\left(\mathbf{Z}^{2^{t-2}-2}\right)^{-} \oplus(\mathbf{Z} / 2 \oplus$ Z) where the last summand is as in 5.10(a).

(c) If the center is $\mathbf{Q}\left(\zeta_{3}, \lambda_{i}\right)$ then the module is $\left(\mathbf{Z}^{2^{i-2}-1}\right)^{-} \oplus \mathbf{Z} / 2$.

Proof. For cases (a) and (b) see Theorem 4.12 of [WA]. To see that case (c) is correct note that the only possiblity of an extra unit is some unit $u$ so that $\bar{u}=-u$. But then $u\left(\zeta_{3}-\left(\zeta_{3}\right)^{-1}\right)$ would be contained in $\mathbf{Q}\left(\lambda_{i}\right)$ and three would ramify there which is impossible. Hence the result follows.

REMARK 5.11. Note that in $U K_{1}^{\prime}\left(\mathbf{Z}\left(1 / 2, \zeta_{3}\right) \pi\right)$ odd index subgroups of the units suffice, and 3.7 shows that the cyclotomic units are such a subgroup, so we can assume the generators in 5.10 are just cyclotomic units.

Section 6 will show that we are able to ignore the effects of the submodules $\left(\mathbf{Z}^{v}\right)^{-}$in 5.10. Hence our interest is focused on the modules

$$
W_{i}=\left(\mathbf{Z} / 2^{i} \oplus \mathbf{Z}\right), \quad \tau(A)=A, \quad \tau(B)=A-B .
$$

LEMMA 5.12. $\widehat{H}_{0}\left(\mathbf{Z} / 2 ; W_{i}\right)=\mathbf{Z} / 2$ with generator $2^{i-1} A$, while $\widehat{H}_{1}\left(\mathbf{Z} / 2 ; W_{i}\right)=0$.

Proof. A resolution of $\mathbf{Z}$ over $\mathbf{Z}(\mathbf{Z} / 2)$ is given by the long exact sequence

$$
\cdots \longrightarrow \mathbf{Z}(\mathbf{Z} / 2) \stackrel{T-1}{\longrightarrow} \mathbf{Z}(\mathbf{Z} / 2) \stackrel{T+1}{\longrightarrow} \mathbf{Z}(\mathbf{Z} / 2) \stackrel{T-1}{\longrightarrow} \mathbf{Z}(\mathbf{Z} / 2) \longrightarrow \cdots
$$

Now tensor the resolution above with $W_{i}$. When we calculate the induced boundary map $\partial$ in the resulting chain complex we have

$$
\begin{array}{cc}
(T+1) A=2 A, & (T+1) B=A, \\
(T-1) A=0, & (T-1) B=A-2 B .
\end{array}
$$

From this 5.12 is a direct calculation. 
Next, let $K=\mathbf{Z} / 6 \times \pi^{\mathrm{ab}}$ be the torsion in $K_{1}^{\prime}\left(\mathbf{Z}\left(\zeta_{3}\right) \pi\right)$. Then we have the diagram
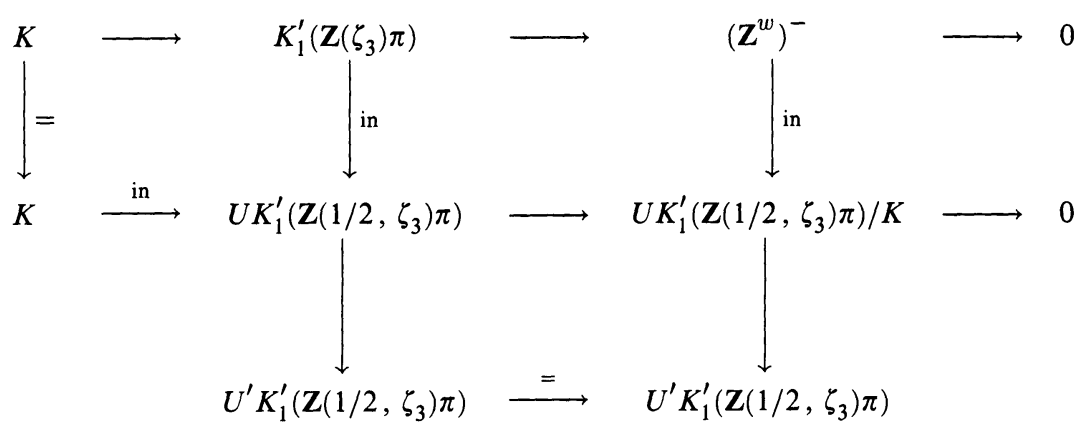

with the two middle columns exact. We are particularly interested in the 3rd column and its homology groups. We have that the groups

$$
\widehat{H}_{*}\left(\mathbf{Z} / 2 ;\left(\mathbf{Z}^{w}\right)^{-}\right)= \begin{cases}(\mathbf{Z} / 2)^{w} & * \equiv 0 \bmod 2, \\ 0 & * \equiv 1 \bmod 2 .\end{cases}
$$

Hence,

$$
\widehat{H}_{0}\left(\mathbf{Z} / 2 ; U K_{1}^{\prime}\left(\mathbf{Z}\left(1 / 2, \zeta_{3}\right) \pi\right) / K\right)
$$

surjects to

$$
\widehat{H}_{0}\left(\mathbf{Z} / 2 ; U K_{1}^{\prime}\left(\mathbf{Z}\left(1 / 2, \zeta_{3}\right) \pi\right)\right),
$$

while the group $\widehat{H}_{1}\left(\mathbf{Z} / 2 ; U K_{1}^{\prime}\left(\mathbf{Z}\left(1 / 2, \zeta_{3}\right) \pi\right) / K\right)$ injects. Thus, we will use the middle horizontal sequence in $(5.13)$ to study the image of $\widehat{H}_{*}(\mathbf{Z} / 2 ; K)$ in $\widehat{H}_{*}\left(\mathbf{Z} / 2 ; U K_{1}^{\prime}\left(\mathbf{Z}\left(1 / 2, \zeta_{3}\right) \pi\right)\right)$. Indeed, we will obtain what we require about the structure of the groups $\widehat{H}_{*}\left(\mathbf{Z} / 2 ; \widetilde{K}_{0}\left(\mathbf{Z}\left(\zeta_{3}\right) \pi\right)\right)$ directly once we have proved

Lemma 5.14. The inclusion $K \rightarrow U K_{1}^{\prime}\left(\mathbf{Z}\left(1 / 2, \zeta_{3}\right) \pi\right)$ induces an injection in homology.

Proof. Let $\mathbf{Z} / 2^{j}$ be a direct summand of $\pi^{\mathrm{ab}}$. Then there is a splitting homomorphism

$$
\phi: \pi^{\mathrm{ab}} \longrightarrow \mathbf{Z} / 2^{j}
$$

and a corresponding sequence of representations

$$
\begin{gathered}
\phi_{i}=\theta_{i} \phi: \pi^{\mathrm{ab}} \longrightarrow \mathbf{Z} / 2^{j} \longrightarrow \mathbf{Q}\left(\zeta_{2^{i}}\right), \\
\left\{\theta_{i}(T)=\zeta_{2^{2}} \in \mathbf{Q}\left(\zeta_{2^{i}}\right)\right\}, \quad 0 \leq i \leq j .
\end{gathered}
$$

In particular note the two extreme representations

$$
\theta_{j}: \mathbf{Z} / 2^{j} \longrightarrow \mathbf{Z}\left(\zeta_{2^{j}}\right), \quad \theta_{1}: \mathbf{Z} / 2^{j} \longrightarrow\{ \pm 1\}
$$


Looking in homology, $\mathbf{Z}\left(\zeta_{2^{\prime}}\right)$ carries the class corresponding to the element $\left\langle 2^{j-1} g\right\rangle$ in $\widehat{H}_{1}\left(\mathbf{Z} / 2 ;\left(\mathbf{Z} / 2^{j}\right)^{+}\right)$while the $\mathbf{Z} / 2$-representation (among others) carries the image of the class $\langle g\rangle$ in $\widehat{H}_{0}\left(\mathbf{Z} / 2 ;\left(\mathbf{Z} / 2^{j}\right)^{+}\right)$, and the result follows.

As indicated in the discussion preceding 5.14, we are now able to present the information we need about the groups

$$
\widehat{H}_{*}\left(\mathbf{Z} / 2 ; U^{\prime} K_{1}^{\prime}\left(\mathbf{Z}\left(1 / 6, \zeta_{3}\right) \pi\right)\right) .
$$

Definition 5.15. Let $q(\pi)$ be the number of irreducible $\mathbf{Q}$ representations of $\pi$, then $n(\pi)=c(\pi)-2 q(\pi)+\lambda(\pi)+d(\pi)$ where $c(\pi)$, $\lambda(\pi)$, and $d(\pi)$ are defined in (3.6).

Using $q(\pi)$ and $n(\pi)$ we have

LEMMA 5.16. Let $U T \pi=U K^{\prime}\left(\mathbf{Z}\left(1 / 2, \zeta_{3}\right) \pi\right) / K$, then

$$
\begin{aligned}
& \widehat{H}_{0}(\mathbf{Z} / 2 ; U T \pi)=\mathbf{Z} / 2^{n(\pi)+q(\pi)-r k\left(\pi^{\mathrm{ab}}\right)-1}, \\
& \widehat{H}_{1}(\mathbf{Z} / 2 ; U T \pi)=\mathbf{Z} / 2^{\lambda(\pi)+d(\pi)-r k\left(\pi^{\mathrm{ab}}\right)-1} .
\end{aligned}
$$

In $\widehat{H}_{0}$ the generators are the $\left\langle v_{i, j}\right\rangle$ and $\langle-1\rangle$ at each maximal subfield in the center of $\mathbf{Q}\left(\zeta_{3}\right) \pi$ where the $v_{i, j}$ run over a basis for units mod squares, while the generators for $\widehat{H}_{1}$ are the $\langle-1\rangle$ 's as we run over all maximal subfields of the center having the form $\mathbf{Q}\left(\zeta_{3}, \lambda_{i}\right)$.

Proof. Note that $c(\pi)$ is just the dimension (over $\mathbf{Q}$ ) of the center of $\mathbf{Q}(\pi)$. Consequently, $c(\pi)-q(\pi)$ is the dimension of the lattice of units (mod torsion) in these centers. In looking at the module $W(\pi)$ we see that the $(\mathbf{Z})^{-}$summands have dimension exactly $n(\pi)$. Likewise, there are exactly $q(\pi)-\lambda(\pi)$ summands $W_{i}$, and the result follows from the previous two lemmas.

As a consequence we have

Corollary 5.17.

$$
\widehat{H}_{0}\left(\mathbf{Z} / 2 ; U^{\prime} K_{1}^{\prime}\left(\mathbf{Z}\left(1 / 2, \zeta_{3}\right) \pi\right)\right)=\mathbf{Z} / 2^{n(\pi)-\alpha(\pi)+q(\pi)-r k\left(\pi^{\mathrm{ab}}\right)-1},
$$

$\widehat{H}_{1}\left(\mathbf{Z} / 2 ; U^{\prime} K_{1}^{\prime}\left(\mathbf{Z}\left(1 / 2, \zeta_{3}\right) \pi\right)\right)=\mathbf{Z} / 2^{\lambda(\pi)+d(\pi)-r k\left(\pi^{\mathrm{ab}}\right)-1+(c(\pi)-q(\pi)-\alpha(\pi))}$

where $\alpha(\pi)$ is the dimension of the kernel of the map on units mod squares induced from the map

$$
U K_{1}^{\prime}\left(\mathbf{Z}\left(1 / 2, \zeta_{3}\right) \pi\right) \longrightarrow U^{\prime} K_{1}^{\prime}\left(\mathbf{Z}\left(1 / 2, \zeta_{3}\right) \pi\right)
$$




\section{COROLlaRY 5.18. The map}

$$
\widehat{H}_{k}\left(\mathbf{Z} / 2 ; U K_{1}^{\prime}\left(\mathbf{Z}\left(1 / 2, \zeta_{3}\right) \pi\right)\right) \stackrel{i_{k}}{\longrightarrow} \widehat{H}_{k}\left(\mathbf{Z} / 2 ; U^{\prime} K_{1}^{\prime}\left(\mathbf{Z}\left(1 / 2, \zeta_{3}\right) \pi\right)\right)
$$

is surjective for $k=0$ (with kernel $\mathbf{Z} / 2^{\alpha(\pi)}$ ), and injective for $k=1$.

The groups $\widehat{H}_{k}\left(\mathbf{Z} / 2 ; \widetilde{K}_{0}\left(\mathbf{Z}\left(\zeta_{3}\right) \pi\right)\right)$ are isomorphic to the groups

$$
\widehat{H}_{k+1}\left(\mathbf{Z} / 2 ; U^{\prime} K_{1}^{\prime}\left(\mathbf{Z}\left(1 / 2, \zeta_{3}\right) \pi\right)\right) \text {, }
$$

and using this isomorphism we identify these groups in subsequent discussions.

We call the cokernel of $i_{1}$ in 5.18 the unit Bochstein group, and the major object of the next section will be to show that this unit Bochstein group, while injecting into $L_{2 k}^{h}\left(\mathbf{Z}\left(\zeta_{3}\right) \pi\right)$, is not in the image of $L_{2 k}^{\prime}\left(\mathbf{Z}\left(\zeta_{3}\right) \pi\right)$ and hence cannot represent non-trivial surgery problems on the image of $\Omega\left(B_{\pi}\right)$.

Likewise, we will show the unit group

$$
i_{0}\left((\mathbf{Z} / 2)^{n(\pi)}\right)=(\mathbf{Z} / 2)^{n(\pi)-\alpha(\pi)}
$$

is in the image of the boundary map from $L_{2 k}^{p}\left(\mathbf{Z}\left(\zeta_{3}\right) \pi\right)$. Hence the only things which will finally matter are the contributions from the $W_{i}$ 's.

However, before we can complete the analysis of $L_{*}^{h}\left(\mathbf{Z}\left(\zeta_{3}\right) \pi\right)$ we must look a little more closely at the groups $U\left(\widehat{\mathbf{Q}}_{2}\left(\zeta_{3}, v_{i}\right)\right)$. The result is

LEMMA 5.19. (a) As a module over the involution $\tau, U \widehat{\mathbf{Q}}_{2}\left(\zeta_{3}\right) \cong$ $\left(\widehat{\mathbf{Z}}_{2}\right)^{2} \times \mathbf{Z} / 2$, with generators $A, B,\langle-1\rangle$ and $\tau A=A+\langle-1\rangle, \tau B=$ $-2 A-B, \tau\langle-1\rangle=\langle-1\rangle$.

(b) As a module over $\tau, U \widehat{\mathbf{Q}}_{2}\left(\zeta_{3}, \lambda_{i}\right) \cong \widehat{\mathbf{Z}}_{2}(\mathbf{Z} / 2)^{2^{i-2}-2} \times U \widehat{\mathbf{Q}}_{2}\left(\zeta_{3}\right)$, where the skew elements in the free summands are 2-adically generated by the cyclotomic units of $\mathbf{Z}\left(\zeta_{3}, \lambda_{i}\right)$.

(c) As a module over $\tau, U \widehat{\mathbf{Q}}_{2}\left(\zeta_{3}, \zeta_{2^{i}}\right) \cong \widehat{\mathbf{Z}}_{2}[\mathbf{Z} / 2]^{2^{i-1}-2} \oplus\left(\widehat{\mathbf{Z}}_{2}\right)^{4} \times \mathbf{Z} / 2^{i}$, where the generators for the non-free summand $V_{i}$ are $A, B, C, D$, and $\left\langle\zeta_{2^{\prime}}\right\rangle=e$, and the involution is given by

$$
\begin{gathered}
\tau e=e, \quad \tau A=2^{i-1} e-A, \quad \tau B=e-B, \\
\tau C=-2 A+C, \quad \tau D=2^{i-2} e-B+D .
\end{gathered}
$$

(d) As a module over $\tau, U \widehat{\mathbf{Q}}_{2}\left(\zeta_{3}, \mu_{i}\right)=\widehat{\mathbf{Z}}_{2}[\mathbf{Z} / 2]^{2^{1-2}-2} \oplus\left(\widehat{\mathbf{Z}}_{2}\right)^{4} \times \mathbf{Z} / 2$, where the generators for the non-free summand $S_{i}$ are $A, B, C^{\prime}, D^{\prime}$ 
and $\langle-1\rangle=e$. The involution is given as in (c) for $A, B, e$, but $\tau C^{\prime}=e-C^{\prime}, \tau D^{\prime}=D^{\prime}-\left(C^{\prime}+A\right)$.

REMARK 5.20. Before outlining the proof of 5.19 we describe some of the generators of the non-free modules. In (a) $A$ can be taken to be $\zeta_{3}-\left(\zeta_{3}\right)^{-1}$, while $B$ must exist to make the homology come out correct. In (b) the generator $A$ is as before, while we can choose $B$ as the unit $\zeta_{3}\left(\zeta_{2^{\prime}}\right)^{-1}+\left(\zeta_{3}\right)^{-1}$ and $C$ is the same as $B$ in 5.19(a). Again, $D$ is just there to make the homology come out correctly. Finally, in (d) $A$ and $B$ are as in $5.19(\mathrm{a})$, while $C$ can be taken as the unit $1-\left(\zeta_{3}\right)^{2} \mu_{i}$, and $D$ is again just there for the homology.

The key to the proof of 5.19 is the norm theorem for non-ramified finite extensions $\mathbf{L}$ of $\mathbf{K}$ where $\mathbf{K}$ is itself a finite extension of $\widehat{\mathbf{Q}}_{2}$. This states that every unit in $\mathbf{K}$ is a norm from $\mathbf{L}$ if the extension is Galois with cyclic Galois group. Since the involution in each case in 5.19 is a Galois automorphism we can apply the norm theorem. It remains to check that the modules are as stated. This is done by reducing mod 2 . This gets the free parts. The specific elements above are then analyzed mod 2 to show they are not squares, and application of Hilbert's Theorem 90 completes the verification of 5.19.

6. The analysis of $L_{*}^{h}\left(Z\left(\zeta_{3}\right) \pi\right)$. Recall (from (3.6)) that $d(\pi)$ is the number of representations having the form $M_{n_{i}}\left(\mathbf{D}_{i}\right)$ where $\mathbf{D}_{i}$ is a quaternion algebra, then we have

THEOREM 6.1. The groups $L_{*}^{h}\left(\mathbf{Z}\left(\zeta_{3}\right) \pi\right)$ are given as follows for $\pi \neq$ 1, a finite 2-group:

$$
\begin{aligned}
& L_{0}^{h}\left(\mathbf{Z}\left(\zeta_{3}\right) \pi\right) \cong \mathbf{Z}^{c(\pi)} \oplus(\mathbf{Z} / 2)^{d(\pi)} \oplus V_{0} . \\
& L_{1}^{h}\left(\mathbf{Z}\left(\zeta_{3}\right) \pi\right) \cong(\mathbf{Z} / 2)^{r k\left(\pi^{\mathrm{ab}}\right)} \oplus V_{1} . \\
& L_{2}^{h}\left(\mathbf{Z}\left(\zeta_{3}\right) \pi\right) \cong \mathbf{Z}^{c(\pi)} \oplus(\mathbf{Z} / 2)^{\lambda(\pi)-r k\left(\pi^{\mathrm{ab}}\right)-d(\pi)-1} \oplus V_{2} . \\
& L_{3}^{h}\left(\mathbf{Z}\left(\zeta_{3}\right) \pi\right) \cong(\mathbf{Z} / 2)^{d(\pi)} .
\end{aligned}
$$

Here, the groups $V_{0}=V_{2}=(\mathbf{Z} / 2)^{n(\pi)-\alpha(\pi)}$ come from the unit Bockstein part of 5.18, and are not in the image of $\Omega_{*}\left(B_{\pi}\right)$. Also, the subgroup

$$
(\mathbf{Z} / 2)^{r k\left(\pi^{\mathrm{ab}}\right)} \text { in } L_{1}()
$$

injects to corresponding elements in $L_{1}^{p}\left(\mathbf{Z} \pi^{\mathrm{ab}}\right)$. The elements in $L_{3}^{h}($ ) come from the $\mathbf{Z} / 2$ 's in $\widehat{H}_{*}\left(\mathbf{Z} / 2 ; \widetilde{K}_{0}\left(\mathbf{Z}\left(\zeta_{3}\right) \pi\right)\right.$ ) associated to the modules $W_{i}$ at the quaternion representations in $\mathbf{Q}\left(\zeta_{3}\right) \pi$. Finally, the elements in $V_{1}$ go to 0 under $\tau_{K}$. 
Proof. We have an effective calculation of $\widehat{H}_{*}\left(\mathbf{Z} / 2 ; \widetilde{K}_{0}\left(\mathbf{Z}\left(\zeta_{3}\right) \pi\right)\right)$ in $\S 5$, and in $\S 2$ and $\S 3$ we calculated $L_{*}^{p}\left(\mathbf{Z}\left(\zeta_{3}\right) \pi\right)$. So we can use the exact sequence in $(2.1)$ to study $L_{*}^{h}\left(\mathbf{Z}\left(\zeta_{3}\right) \pi\right)$.

Note that the map

$$
L_{\mathrm{ev}}^{p}\left(\mathbf{Z}\left(\zeta_{3}\right) \pi\right) \longrightarrow \widehat{H}_{1}\left(\mathbf{Z} / 2 ; \widetilde{K}_{0}\left(\mathbf{Z}\left(\zeta_{3}\right) \pi\right)\right)
$$

is given on generators as follows: if $\gamma$ represents the discriminant of the form

$$
\left\langle\gamma_{1}\right\rangle \perp\left\langle\gamma_{2}\right\rangle \perp \cdots \perp\left\langle\gamma_{n}\right\rangle
$$

then $\gamma$ is a unit in $K_{1}\left(\widehat{\mathbf{Q}}_{2}\left(\zeta_{3}\right) \pi\right)$ fixed under the involution $(\alpha \leftrightarrow$ $\left.\alpha^{-*}\right)$. Moreover $\gamma$ is the image of a class $\beta \in K_{1}\left(\mathbf{Z}\left(\zeta_{3}\right) \pi\right)$. Consequently there is an element $\delta \gamma \in K_{1}\left(\widehat{\mathbf{Q}}_{2}\left(\zeta_{3}\right) \pi\right)$ so that $(\delta \gamma)(\delta \gamma)^{-*}=\gamma$. Then $\{\delta \gamma\} \in \widetilde{K}_{0}\left(\mathbf{Z}\left(\zeta_{3}\right) \pi\right)$ represents a well defined class $\mu(\gamma)$ in $\widehat{H}_{1}\left(\mathbf{Z} / 2 ; \widetilde{K}_{0}\left(\mathbf{Z}\left(\zeta_{3}\right) \pi\right)\right)$ and we have

$$
\partial\left(\left\langle\gamma_{1}\right\rangle \perp\left\langle\gamma_{2}\right\rangle \perp \cdots \perp\left\langle\gamma_{n}\right\rangle\right)=\mu(\gamma)
$$

(See e.g. the discussion in [H-M], in particular Lemma 5.4 there and its proof.) This shows that every class in $\widehat{H}_{1}\left(\mathbf{Z} / 2 ; \widetilde{K}_{0}\left(\mathbf{Z}\left(\zeta_{3}\right) \pi\right)\right)$ is in the image of $\partial$ except the classes corresponding to $\langle-1\rangle$ at type $\mathrm{IIb}$ representations in the map

$$
\partial: L_{0}^{p}\left(\mathbf{Z}\left(\zeta_{3}\right) \pi\right) \longrightarrow \widehat{H}_{1}\left(\mathbf{Z} / 2 ; \widetilde{K}_{0}\left(\mathbf{Z}\left(\zeta_{3}\right) \pi\right)\right) .
$$

(The classes in $K_{1}\left(\widehat{\mathbf{Q}}_{2}\left(\zeta_{3}\right) \pi\right)$ corresponding to -1 at the relevant representations are represented by $\left\langle\zeta_{3}-\left(\zeta_{3}\right)^{-1}\right\rangle$ at those same representations. For more details see the discussion from 5.18 to 5.20.) Likewise the situation reverses in the map

$$
\partial: L_{2}^{p}\left(\mathbf{Z}\left(\zeta_{3}\right) \pi\right) \longrightarrow \widehat{H}_{1}\left(\mathbf{Z} / 2 ; \widetilde{K}_{0}\left(\mathbf{Z}\left(\zeta_{3}\right) \pi\right)\right) .
$$

and we obtain that the $\langle-1\rangle$ 's at the $\lambda(\pi)$ representations which are not quaternion give rise to the cokernel of $\partial$. The situation is not much different for

$$
\partial_{2 k+1}: L_{2 k+1}^{p}\left(\mathbf{Z}\left(\zeta_{3}\right) \pi\right) \longrightarrow \widehat{H}_{0}\left(\mathbf{Z} / 2 ; \widetilde{K}_{0}\left(\mathbf{Z}\left(\zeta_{3}\right) \pi\right)\right) .
$$

Here it is easily seen that the $\mathbf{Z} / 2$ 's in $\left.L_{2 k+1}^{p}\left(\mathbf{Z}\left(\zeta_{3}\right) \pi\right)\right)$ have image the class $\left\{\zeta_{3}-\left(\zeta_{3}\right)^{-1}\right\}$ at the appropriate representation. Hence, $\partial_{3}$ is injective, while $\partial_{1}$ surjects onto the

$$
\mathbf{Z} / 2^{\lambda(\pi)-r k\left(\pi^{\mathrm{ab}}\right)-1} \text { part of } \mathbf{Z} / 2^{\lambda(\pi)+d(\pi)-r k\left(\pi^{\mathrm{ab}}\right)-1}
$$

in $\widehat{H}_{0}$ with kernel $\mathbf{Z} / 2^{r k\left(\pi^{a b}\right)}$ and cokernel $\mathbf{Z} / 2^{d(\pi)}$. In both cases the unit Bockstein part is not in the image of $\partial_{2 k+1}$ and hence maps into 
$L_{2 k}^{h}\left(\mathbf{Z}\left(\zeta_{3}\right) \pi\right)$. This completes the first step in the proof of 6.1 , the explicit determination of $L_{k}^{h}\left(\mathbf{Z}\left(\zeta_{3}\right) \pi\right)$.

We now have to make two further calculations. First we must show that the elements in $V_{1}$ map trivially under $\tau_{K}$. But since $V_{1}$ is in the image of $\widehat{H}_{0}\left(\mathbf{Z} / 2 ; \widetilde{K}_{0}\left(\mathbf{Z}\left(\zeta_{3}\right) \pi\right)\right)$ we can use the results of $\S 4$. In particular, note that the elements in $V_{1}$ come from the $\widehat{\mathbf{Q}}_{2}\left(\zeta_{3}, \lambda_{i}\right)$ parts of $K_{1}\left(\widehat{\mathbf{Q}}_{2}\left(\zeta_{3}\right) \pi\right)$, and so the Norm map under the Galois automorphism $\zeta_{3} \leftrightarrow \zeta_{3}^{-1}$ is identified with the Norm map under the involution. But the representatives for $V_{1}$ all have norm -1 under the involution, and so the $\langle-1\rangle$ at the corresponding representations give the image of $V_{1}$. The elements surviving to $V_{1}$ all come from representations

$$
\widehat{\mathbf{Q}}_{2}\left(\zeta_{3}\right) \otimes M_{n_{i}}\left(\mathbf{Q}\left(\lambda_{i}\right)\right) \text {, }
$$

and $\langle-1\rangle$ for the corresponding representation $M_{n_{i}}\left(\mathbf{Q}\left(\lambda_{i}\right)\right)$ is the image of a global unit, hence represents 0 in $\widetilde{K}_{0}(\mathbf{Z} \pi)$. On the other hand the generators for the $(\mathbf{Z} / 2)^{d(\pi)}$ in $L_{3}^{h}\left(\mathbf{Z}\left(\zeta_{3}\right) \pi\right)$ all come from $\langle-1\rangle$ 's at the representations $\widehat{\mathbf{Q}}_{2}\left(\zeta_{3}\right) \otimes M_{n_{i}}\left(\mathbf{D}_{i}\right)$ with $\mathbf{D}_{i}$ a non-trivial quaternion algebra. These elements are not a priori 0 in $\widetilde{K}_{0}(\mathbf{Z} \pi)$.

The final step in the proof is to handle the unit Bochstein classes. For this we find it convenient to use Wall's intermediate $L$-groups [W3] which he denotes $L_{k}^{\prime}\left(\mathbf{Z}\left(\zeta_{3}\right) \pi\right)$. Recall (5.3) that $K_{1}^{\prime}\left(\mathbf{Z}\left(\zeta_{3}\right) \pi\right)$ is the image of $K_{1}\left(\mathbf{Z}\left(\zeta_{3}\right) \pi\right)$ in $K_{1}\left(\widehat{\mathbf{Q}}_{2}\left(\zeta_{3}\right) \pi\right)$ under the usual inclusion. Also,

$$
\mathrm{Wh}^{\prime}\left(\mathbf{Z}\left(\zeta_{3}\right) \pi\right)=K_{1}^{\prime}\left(\mathbf{Z}\left(\zeta_{3}\right) \pi\right) /(\text { torsion })=\left(\mathbf{Z}^{w}\right)^{-}
$$

is the group at the top of the third column in (5.13). There is an exact sequence

$$
\begin{aligned}
\cdots \rightarrow \widehat{H}_{k}\left(\mathbf{Z} / 2 ; \mathrm{Wh}^{\prime}\left(\mathbf{Z}\left(\zeta_{3}\right) \pi\right)\right) & \stackrel{\partial}{\rightarrow} L_{k}^{\prime}\left(\mathbf{Z}\left(\zeta_{3}\right) \pi\right) \rightarrow L_{k}^{h}\left(\mathbf{Z}\left(\zeta_{3}\right) \pi\right) \\
& \left.\stackrel{b}{\rightarrow} \widehat{H}_{k-1}\left(\mathbf{Z} / 2 ; \mathrm{Wh}^{\prime}\left(\zeta_{3}\right) \pi\right)\right) \stackrel{\partial}{\rightarrow} \cdots
\end{aligned}
$$

and the map $L_{k}^{s}\left(\mathbf{Z}\left(\zeta_{3}\right) \pi\right) \rightarrow L_{k}^{h}\left(\mathbf{Z}\left(\zeta_{3}\right) \pi\right)$ factors through $L_{k}^{\prime}\left(\mathbf{Z}\left(\zeta_{3}\right) \pi\right)$. (In (6.3), to be more consistent with the $\widetilde{K}_{0}$ involution we choose the involution $\alpha \leftrightarrow\left(\bar{\alpha}^{-1}\right)$ on $K_{1}^{\prime}$, rather than the more usual $\alpha \leftrightarrow \bar{\alpha}$, which explains the dimensions of the homology groups.)

The proof of 6.1 will be complete when we have shown that the map

$$
b: L_{k}^{h}\left(\mathbf{Z}\left(\zeta_{3}\right) \pi\right) \longrightarrow \widehat{H}_{k}\left(\mathbf{Z} / 2 ; \operatorname{Wh}^{\prime}\left(\mathbf{Z}\left(\zeta_{3}\right) \pi\right)\right)
$$

in (6.3) is injective on $V_{k}$ for $k$ even. 
To begin note the projection

$$
p: K_{1}^{\prime}\left(\mathbf{Z}\left(\zeta_{3}\right) \pi\right) \longrightarrow \mathrm{Wh}^{\prime}\left(\mathbf{Z}\left(\zeta_{3}\right) \pi\right)
$$

inducing the map

$$
p_{*}: \widehat{H}_{*}\left(\mathbf{Z} / 2 ; K_{1}^{\prime}\left(\mathbf{Z}\left(\zeta_{3}\right) \pi\right)\right) \longrightarrow \widehat{H}_{*}\left(\mathbf{Z} / 2 ; \mathrm{Wh}^{\prime}\left(\mathbf{Z}\left(\zeta_{3}\right) \pi\right)\right) \text {. }
$$

From (5.13), 5.14 we see that $\widehat{H}_{*}\left(\mathbf{Z} / 2 ; K_{1}^{\prime}\left(\mathbf{Z}\left(\zeta_{3}\right) \pi\right)\right)=\widehat{H}_{*}(\mathbf{Z} / 2 ; K) \oplus$ $B$, and $p_{*}$ identifies $B$ with $\widehat{H}_{*}\left(\mathbf{Z} / 2 ; \mathrm{Wh}^{\prime}\left(\mathbf{Z}\left(\zeta_{3}\right) \pi\right)\right)$. We also have the short exact sequence

$$
0 \rightarrow K_{1}^{\prime}\left(\mathbf{Z}\left(\zeta_{3}\right) \pi\right) \rightarrow U K_{1}^{\prime}\left(\mathbf{Z}\left(1 / 2, \zeta_{3}\right) \pi\right) \rightarrow U^{\prime} K_{1}^{\prime}\left(\mathbf{Z}\left(\zeta_{3}\right) \pi\right) \rightarrow 0
$$

which gives rise to the long exact sequence

$$
\begin{aligned}
\cdots & \longrightarrow \widehat{H}_{k}\left(\mathbf{Z} / 2 ; K_{1}^{\prime}\left(\mathbf{Z}\left(\zeta_{3}\right) \pi\right)\right) \longrightarrow \widehat{H}_{k}\left(\mathbf{Z} / 2 ; U K_{1}^{\prime}\left(\mathbf{Z}\left(1 / 2, \zeta_{3}\right) \pi\right)\right) \\
& \longrightarrow \widehat{H}_{k}\left(\mathbf{Z} / 2 ; U^{\prime} K_{1}^{\prime}\left(\mathbf{Z}\left(\zeta_{3}\right) \pi\right)\right) \stackrel{\partial}{\longrightarrow} \widehat{H}_{k-1}\left(\mathbf{Z} / 2 ; K_{1}^{\prime}\left(\mathbf{Z}\left(\zeta_{3}\right) \pi\right)\right) \longrightarrow \cdots .
\end{aligned}
$$

Finally, recall the isomorphism (5.10)

$$
\widehat{H}_{k}\left(\mathbf{Z} / 2 ; \widetilde{K}_{0}\left(\mathbf{Z}\left(\zeta_{3}\right) \pi\right)\right) \stackrel{\partial^{\prime}}{\longrightarrow} \widehat{H}_{k-1}\left(\mathbf{Z} / 2 ; U^{\prime} K_{1}^{\prime}\left(\mathbf{Z}\left(\zeta_{3}\right) \pi\right)\right)
$$

induced from the exact sequence

$$
0 \rightarrow U^{\prime} K_{1}^{\prime}\left(\mathbf{Z}\left(\zeta_{3}\right) \pi\right) \rightarrow U K_{1}\left(\widehat{\mathbf{Q}}_{2}\left(\zeta_{3}\right) \pi\right) / K_{1}^{\prime}\left(\widehat{\mathbf{Z}}_{2}\left(\zeta_{3}\right) \pi\right) \rightarrow \widetilde{K}_{0}\left(\mathbf{Z}\left(\zeta_{3}\right) \pi\right) \rightarrow 0 .
$$

LEMMA 6.7. There is a commutative diagram

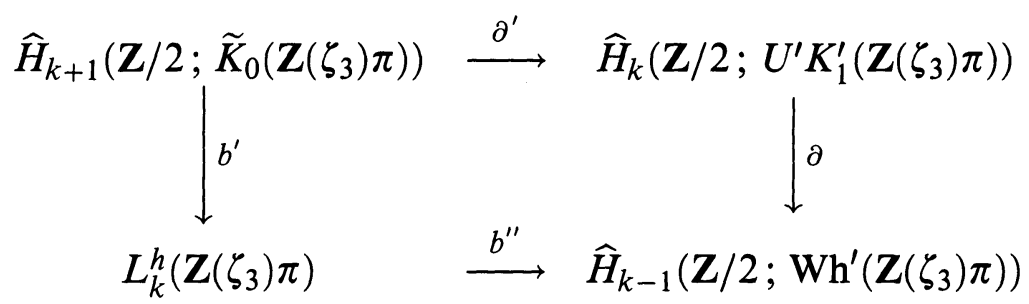

where $b=p_{*} b^{\prime \prime}$ is the map in the sequence (6.3), $b^{\prime}$ is the associated map for the sequence

$$
\cdots L^{p} \rightarrow \widehat{H} \stackrel{b^{\prime}}{\rightarrow} L^{h} \rightarrow L^{p} \ldots,
$$

$\partial$ is the map in (6.5), and $\partial^{\prime}$ is the map in (6.6).

REMARK 6.8. 6.7 is (probably) folklore, but, as far as I know, there is no proof in the literature, so we now indicate how the proof proceeds. The first step is to note that in our case only $k$ even matters as $\partial^{\prime}$ is 0 when $k$ is odd. But here $b^{\prime}(\{P\})$ is represented by the obvious plus or minus symmetric pairing $\alpha$ on $P \oplus P^{*}$, and $P \oplus P^{*}$ is free. 
Now, $\alpha$ becomes a non-trivial matrix when we choose a free basis for $P \oplus P^{*}$, and we need to calculate its discriminant. The module $P$ is given as a pull back as follows

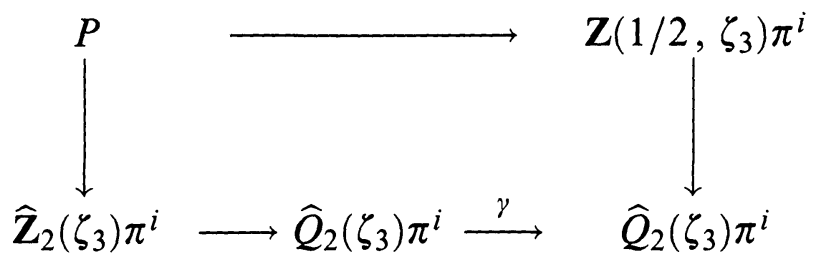

where $\gamma$ is an appropriate isomorphism, and the other arrows are the usual inclusions.

The conditions imply that $\{\gamma\}$ represents the class of $P$ in $K_{1}\left(\widehat{\mathbf{Q}}_{2}\left(\zeta_{3}\right) \pi\right),\{\gamma\}^{2}$ is the image of some class from $K_{1}\left(\mathbf{Z}\left(\zeta_{3}\right) \pi\right)$, and $\left\{\gamma \gamma^{-*}\right\}$ is in the image from $K_{1}^{\prime}\left(\mathbf{Z}\left(1 / 2, \zeta_{3}\right) \pi\right)$. Thus the same is true at least up to commutators for the matrices

$$
\left(\begin{array}{cc}
1 & 0 \\
0 & \gamma^{2}
\end{array}\right)=A \text { and }\left(\begin{array}{cc}
1 & 0 \\
0 & \gamma\left(\gamma^{-*}\right)
\end{array}\right)=B
$$

Now consider the diagram

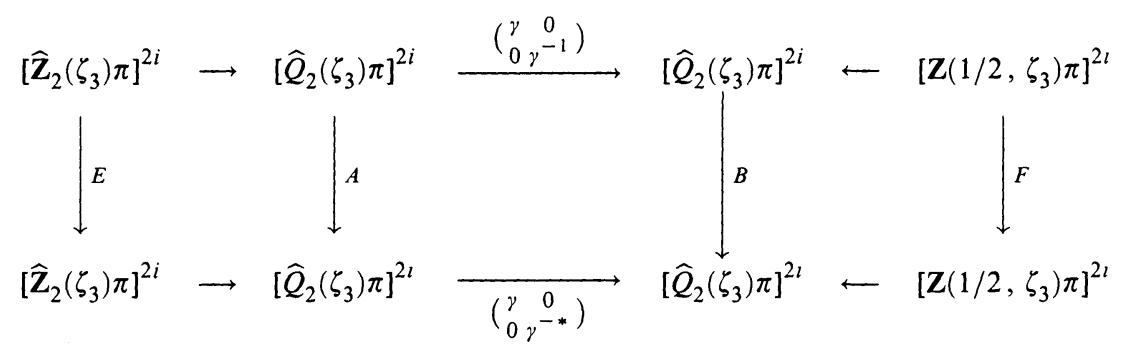

The top row defines the free module $\mathbf{Z}\left(\zeta_{3}\right) \pi^{2 i}$ since the matrix

$$
\left(\begin{array}{cc}
\gamma & 0 \\
0 & \gamma^{-1}
\end{array}\right)
$$

is a commutator, and by the remarks $E$ and $F$ above exist, and the diagram commutes up to some commutators. But the bottom row defines the module $P \oplus P^{*}$, and we have the diagram

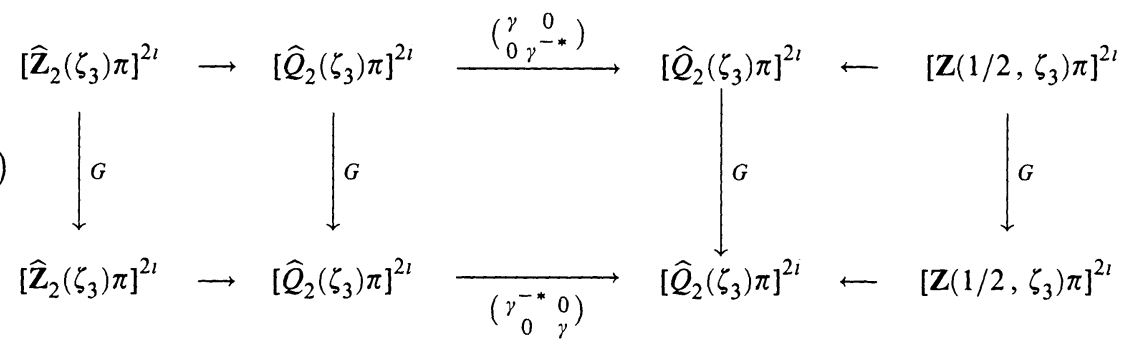


where $G$ is the matrix

$$
\left(\begin{array}{ll}
0 & 1 \\
\varepsilon & 0
\end{array}\right)
$$

and $\varepsilon$ is \pm 1 . This induces the evident dual pairing on $P \oplus P^{*}$, and when followed by the dual of (6.9) gives the associated map

$$
\alpha: \mathbf{Z}\left(\zeta_{3}\right) \pi^{2 i} \rightarrow\left(\mathbf{Z}\left(\zeta_{3}\right) \pi^{2 i}\right)^{*} .
$$

But in view of this, $\{\alpha\}$ in $K_{1}^{\prime}\left(\mathbf{Z}\left(1 / 2, \zeta_{3}\right) \pi\right)$ is $\left\{B B^{*}\right\}=\left\{A^{2}\right\}$, and this implies Lemma 6.7.

REMARK 6.11. 6.7 takes care of the differentials for all elements in $L_{2 k}^{h}\left(\mathbf{Z}\left(\zeta_{3}\right) \pi\right)$ which are associated to non-trivially truncated elements in $U^{\prime} K_{1}^{\prime}\left(Z\left(\zeta_{3}\right) \pi\right)$. The remaining elements (associated to the $\alpha(\pi)$ in $5.17,6.1)$ are in the image of torsion free classes mapping to generators in $L_{2 k}^{p}\left(\mathbf{Z}\left(\zeta_{3}\right) \pi\right)$. This follows from a straightforward modification of the proof of 6.7. (See also $\S 2$ in [M4].) In particular $b$ in (6.3) is surjective. However only the torsion classes of $L_{2 k}^{h}\left(\mathbf{Z}\left(\zeta_{3}\right) \pi\right)$ matter in studying $\tau_{K}$.

6.1 is now a direct consequence.

This completes the $L$-group calculations.

REMARK 6.12. 6.1 is already enough to establish our main results in odd dimensions, since the classes in $L_{3}^{h}\left(\mathbf{Z}\left(\zeta_{3}\right) \pi\right)$ are evidently associated to induced images from quaternion subquotients of $\pi$ of the classes in $L_{h}^{3}(Z \pi)$ associated to the spheres $S^{8 k+3}$ with the usual quaternion actions. Of course, we should emphasize that not all of these classes can actually occur as surgery obstructions. The discussion in $\S 7$ will clarify this.

The generators in $L_{1}^{h}\left(\mathbf{Z}\left(\zeta_{3}\right) \pi\right)$ are associated to the images of certain lens spaces obtained from mapping $Z / 2^{j}$ into $\pi$ to account for each $\mathbf{Z} / 2^{k}$ in $\pi^{\mathrm{ab}}$. Explicitly, choose a generator $\gamma$ for $\pi^{\mathrm{ab}}$. Then there is a $\beta$ in $\pi$ with image $\gamma$, and $\beta^{2^{v}}=1$ for some sufficiently large $d$. Thus, we have the diagram

$$
\mathbf{Z} / 2^{v} \longrightarrow \pi \longrightarrow \mathbf{Z} / 2^{i}
$$

and the composite is surjective. The map of classifying spaces is the one referred to above.

This case differs from the situation for the quaternion groups since

$$
H_{1}(\pi ; \mathbf{Z}) \rightarrow H_{1}\left(\pi^{\mathrm{ab}} ; \mathbf{Z}\right)
$$

is an isomorphism for a finite 2-group $\pi$. Because each generator $\alpha \epsilon$ $H_{1}(\pi ; \mathbf{Z} / 2)$ can be represented by a map $\lambda_{\alpha}: S^{1} \rightarrow B_{\pi}$, we see that the 
map is also an isomorphism in oriented bordism $\Omega_{1}\left(B_{\pi}\right) \rightarrow \Omega_{1}\left(B_{\pi^{\mathrm{ab}}}\right)$. But for an abelian 2 group it follows from Wall's original result, [W1], that the surgery obstruction map $\left\{(M, f) \in \Omega_{1}\left(B_{\pi}\right)\right\} \mapsto \sigma_{f}(\mathrm{id} \times \sigma)$ gives at least an injection $\Omega_{1}\left(B_{\pi}\right) \otimes \mathbf{Z} / 2 \rightarrow L_{3}^{p}(\mathbf{Z} \pi)$. On the other hand, 6.1 shows that the image can be no bigger than this. We have thus proved

COROLlARY 6.13. The image of the surgery obstructions $\sigma_{f}(\mathrm{id} \times \sigma)$ in $L_{3}^{h}(\mathbf{Z} \pi)$ is isomorphic to $H_{1}(\pi ; \mathbf{Z}) \otimes \mathbf{Z} / 2$ for any finite group $\pi$.

REMARK 6.14. In $L_{2 k}^{h}\left(\mathbf{Z}\left(\zeta_{3}\right) \pi\right)$ the torsion free elements must be in the image of the torsion free part of $\Omega_{2 k}\left(B_{\pi}\right)$, and since $\pi$ is finite we have that $\Omega_{4 i}\left(B_{\pi}\right)=\mathbf{Z}^{w} \oplus$ torsion $\}$ with the $\mathbf{Z}^{w}$ coming from $\Omega_{4 i}(p t)$ while $\Omega_{4 k i+2}\left(B_{\pi}\right)=$ \{torsion . But things coming from $\Omega_{4 i}(p t)$ factor through the usual simply connected product formula for which only the index matters. Thus, the only torsion free class which can be in the image of bordism is the class $\langle 1\rangle$. This is represented by the complex projective space $C P^{2 i}$, and by the simply connected product formula, ([W1] Lemma 13B.4, p.177), the product of $\mathbf{C} P^{2 n}$ with the Kervaire problem is non-trivial, so $\tau_{K}(\langle 1\rangle)=$ usual Kervaire class is the only geometrically non-trivial class in dimensions congruent to 2 mod 4 coming from torsion free elements.

Similarly, the torsion free part of $L_{2}^{h}\left(\mathbf{Z}\left(\zeta_{3}\right) \pi\right)$ cannot be geometrically interesting.

7. Explicit calculations for dihedral groups when $k=2$. At the end of $\S 6$ we finished the discussion of the possible surgery obstructions for odd dimensional products with the Kervaire problem. Additionally, for the even groups $L_{2 k}^{h}\left(\mathbf{Z}\left(\zeta_{3}\right) \pi\right), 6.1$ shows that the elements in $V_{0}$, $V_{2}$, cannot be in the image from $\Omega_{*}\left(B_{\pi}\right)$. But more is true. Since $\Omega_{4 k+2}\left(B_{\pi}\right)$ is torsion, and $\Omega_{4 k}\left(B_{\pi}\right)=\Omega_{4 k}(p t) \oplus$ torsion, we see that

$$
\begin{aligned}
\operatorname{im}\left(\boldsymbol{\Omega}_{4 k}\left(B_{\pi}\right)\right) & \subset L_{4 k}\left(\mathbf{Z}\left(\zeta_{3}\right),{ }^{-}\right) \oplus \text { torsion } \\
& =\mathbf{Z} \oplus(\mathbf{Z} / 2)^{d(\pi)} \subset L_{4 k}^{h}\left(\mathbf{Z}\left(\zeta_{3}\right) \pi,{ }^{-}\right),
\end{aligned}
$$

while

$$
\operatorname{im}\left(\Omega_{4 k+2}\left(B_{\pi}\right)\right) \subset(\mathbf{Z} / 2)^{\lambda(\pi)-r k\left(\pi^{\mathrm{ab}}\right)-d(\pi)-1} .
$$

In this section we calculate on model groups to determine the image in (7.2), and in $\S 8$ we will determine the image for (7.1). In fact, in view of the history of the elements in (7.2), (the fact that they project non-trivially to $\pi^{\mathrm{ab}}$ or are induced up from dihedral subquotients), 
we need only study (7.2) for the groups $\mathbf{Z} / 2 \oplus \mathbf{Z} / 2$, and the dihedral groups

$$
D_{2^{i+1}}=\left\{x, y \mid x^{2^{i}}=y^{2}=(x y)^{2}=1\right\} .
$$

This reduction is formalized and discussed at length in $\S 2$ of [M4], but it just amounts to realizing that restriction to subgroups and then projection to quotients will detect all the elements in the subgroups described in (7.1), (7.2). Consequently, these elements are in the image of $\Omega_{2 *}\left(B_{\pi}\right)$ only if the corresponding elements for the subquotients are in the image.

Of course, this condition is not sufficient. If $N \stackrel{\phi}{\leftarrow} H \hookrightarrow \pi$ gives a key representation by inducing up from the model representation on $N$, then the map

$$
L_{2 i}^{h}\left(\mathbf{Z}\left(\zeta_{3}\right) \pi\right) \stackrel{\text { res }}{\longrightarrow} L_{2 i}^{h}\left(\mathbf{Z}\left(\zeta_{3}\right) \pi\right) \longrightarrow L_{2 i}^{h}\left(\mathbf{Z}\left(\zeta_{3}\right) \pi\right)
$$

is non-trivial on the associated class in $L_{2 i}^{h}\left(\mathbf{Z}\left(\zeta_{3}\right) \pi\right)$. Moreover, as we run over all these maps for the various representations of the given type, the maps are independent, hence, taken together, they detect all the classes of this type in $L_{2 i}^{h}\left(Z\left(\zeta_{3}\right) \pi\right)$.

Let $\operatorname{tr}: \Sigma^{0} B_{\pi} \rightarrow \Sigma^{0} B_{H}$ be the stable transfer map. Then the following diagram commutes:

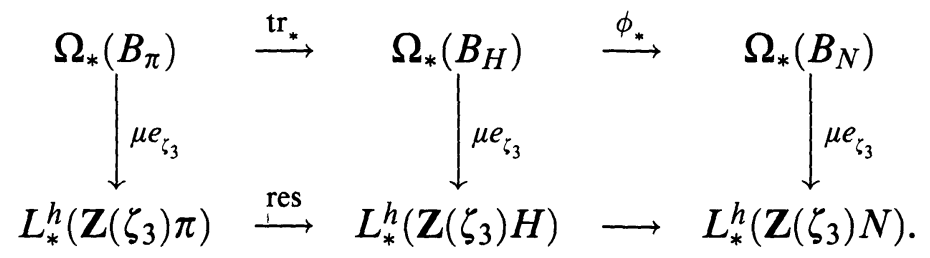

(Recall the discussion of $e_{\lambda}$ in the introduction, $\S 1$, and of $\mu$ in 1.12.) Consequently, if $\left(\mu e_{\zeta_{3}}\right)$ is known on the model groups then it is determined on $\Omega_{2 *}\left(B_{\pi}\right)$ from 7.3.

These questions will be considered in more detail in Part II. Now we begin the explicit calculations by considering the group $\mathbf{Z} / 2 \times \mathbf{Z} / 2$.

LEMMA 7.4. $\widetilde{K}_{0}\left(\mathbf{Z}\left(\zeta_{3}\right) \mathbf{Z} / 2 \times \mathbf{Z} / 2\right)=\mathbf{Z} / 2$ with generator given as the image of the class

$$
\left\{1,1,1,1+2 \zeta_{3}\right\} \in K_{1}\left(\widehat{Q}_{2}\left(\zeta_{3}\right) \mathbf{Z} / 2 \times \mathbf{Z} / 2\right)
$$

under the map $\partial$ in the Mayer-Vietoris sequence of (5.1) where the coefficients are the corresponding units at the four representations ++ , ,,+--+ and -- . 
Proof. Set $\pi=\mathbf{Z} / 2 \times \mathbf{Z} / 2=\left\{x, y \mid x^{2}=y^{2}=(x y)^{2}=1\right\}$. We know [W4] that

$$
\begin{aligned}
K_{1}\left(\widehat{\mathbf{Z}}_{2}\left(\zeta_{3}\right) \pi\right) & =\mathbf{Z} / 6 \times \pi^{\mathrm{ab}} \times \mathrm{Wh}\left(\widehat{\mathbf{Z}}_{2}\left(\zeta_{3}\right) \pi\right) \\
& =\mathbf{Z} / 6 \times(\mathbf{Z} / 2 \times \mathbf{Z} / 2) \times\left(\widehat{\mathbf{Z}}_{2}^{+}\right)^{8},
\end{aligned}
$$

and the elements $-\zeta_{3}, x, y$ generate the torsion part. To make the calculation required for 7.4 we need explicit generators for the torsion free part as well. To this end we consider $W=K_{1}\left(\widehat{\mathbf{Z}}_{2}\left(\zeta_{3}\right) /(8)(\pi)\right)$, and the filtration

$$
U_{2} \subset U_{1} \subset W
$$

where

$$
U_{i}=\operatorname{Ker}\left\{W \longrightarrow K_{1}\left(\widehat{\mathbf{Z}}_{2}\left(\zeta_{3}\right) /\left(2^{i}\right)(\pi)\right)\right\} .
$$

It is direct that

$$
U_{2} \cong F_{4}(\mathbf{Z} / 2 \times \mathbf{Z} / 2)^{+}
$$

with generators $5,1+4 \zeta_{3}, 1+4(x-1), 1+4 \zeta_{3}(x-1), 1+4(y-1)$, $1+4 \zeta_{3}(y-1), 1+4(x-1)(y-1)$, and $1+4 \zeta_{3}(x-1)(y-1)$. Similarly,

$$
U_{1} / U_{2} \cong F_{4}(\mathbf{Z} / 2 \times \mathbf{Z} / 2)^{+}
$$

with generators $-1,1+2 \zeta_{3}, 1+2(x-1), 1+2 \zeta_{3}(x-1)$, etc.. When we square these generators in $W$ we see that all the generators for $U_{2}$ above are accounted for except $1+4 \zeta_{3}$. Finally,

$$
W / U_{1} \cong K_{1}\left(F_{4}(\mathbf{Z} / 2 \times \mathbf{Z} / 2)\right),
$$

and has generators $\zeta_{3}, x, y, 1+\zeta_{3}(x-1), 1+\zeta_{3}(y-1), 1+$ $(x-1)(y-1)$, and $1+\zeta_{3}(x-1)(y-1)$. Squaring the non-torsion generators we see that 4 of the generators for $U_{1}$ are redundant, and a set of torsion free generators in $K_{1}\left(\widehat{\mathbf{Z}}_{2}\left(\zeta_{3}\right) \mathbf{Z} / 2 \times \mathbf{Z} / 2\right)$ are given as the elements $1+\zeta_{3}(x-1), 1+\zeta_{3}(y-1), 1+\zeta_{3}(x-1)(y-1)$, $1+(x-1)(y-1), 1+2 \zeta_{3}, 1+2 \zeta_{3}(x-1), 1+2 \zeta_{3}(y-1)$ and $1+4 \zeta_{3}$. Next note that

$$
U K_{1}\left(\widehat{\mathbf{Q}}_{2}\left(\zeta_{3}\right) \mathbf{Z} / 2 \times \mathbf{Z} / 2\right) \cong\left\{U \widehat{\mathbf{Q}}_{2}\left(\zeta_{3}\right)\right\}^{4} \cong\left\{\mathbf{Z} / 6 \times \widehat{\mathbf{Z}}_{2}^{+} \times \widehat{\mathbf{Z}}_{2}^{+}\right\}^{4}
$$

The generators above for $K_{1}\left(\widehat{\mathbf{Z}}_{2}\left(\zeta_{3}\right) \mathbf{Z} / 2 \times \mathbf{Z} / 2\right)$ map into these units via the correspondence

$$
x \longrightarrow(1,-1,1,-1), y \longrightarrow(1,1,-1,-1), x y \longrightarrow(1,-1,-1,1) \text {. }
$$

Hence a direct calculation shows

$$
U K_{1}\left(\widehat{\mathbf{Q}}_{2}\left(\zeta_{3}\right) \pi\right) / K_{1}\left(\widehat{\mathbf{Z}}_{2}\left(\zeta_{3}\right) \pi\right)_{(2)}=\mathbf{Z} / 2 \times \mathbf{Z} / 2
$$


with generators $\{1,1,1,-1\}$ and $\left\{1,1,1,1+2 \zeta_{3}\right\}$. But the image of the global units

$$
U K_{1}^{\prime}\left(\mathbf{Z}\left(1 / 2, \zeta_{3}\right) \pi\right)=(\mathbf{Z} / 6)^{4}
$$

is evidently the equivalence class of the element $\{1,1,1,-1\}$ and 7.4 follows.

It should be noted at this point that $\widetilde{K}_{0}(\mathbf{Z}(\mathbf{Z} / 2 \times \mathbf{Z} / 2))=0$. This has the effect in $L_{2 *}^{h}(\mathbf{Z}(\mathbf{Z} / 2 \times \mathbf{Z} / 2))$, of making certain elements which should have been present actually equal to 0 , and was the core of the problem with previous attempts to understand the possible surgery obstructions for closed manifolds. It is precisely because $\widetilde{K}_{0}\left(\mathbf{Z}\left(\zeta_{3}\right) \pi\right)$ contains elements such as the $Z / 2$ in 7.4 , that the current approach works.

COROLlaRY 7.5. (a) $L_{1}^{h}\left(\mathbf{Z}\left(\zeta_{3}\right) \mathbf{Z} / 2 \times \mathbf{Z} / 2\right)=(\mathbf{Z} / 2)^{3}$, with image in $L_{1}^{p}\left(\mathbf{Z}\left(\zeta_{3}\right) \mathbf{Z} / 2 \times \mathbf{Z} / 2\right)$ a copy of $(\mathbf{Z} / 2)^{2}$.

(b) $L_{0}^{h}\left(\mathbf{Z}\left(\zeta_{3}\right) \mathbf{Z} / 2 \times \mathbf{Z} / 2\right)=\mathbf{Z}^{4}$ injects into $L_{0}^{p}\left(\mathbf{Z}\left(\zeta_{3}\right) \mathbf{Z} / 2 \times \mathbf{Z} / 2\right)=$ $\mathbf{Z}^{4}$ with $\mathbf{Z} / 2$ cokernel. An explicit basis for this latter group is

$$
\langle 1,1,1,1\rangle,\langle 1,-1,1,1\rangle,\langle 1,1,-1,1\rangle \text {, and }\langle 1,1,1,-1\rangle \text {. }
$$

Each of these last three elements maps onto the $\mathbf{Z} / 2$ cokernel.

(c) $L_{3}^{h}\left(\mathbf{Z}\left(\zeta_{3}\right) \mathbf{Z} / 2 \times \mathbf{Z} / 2\right)=0$.

(d) $L_{2}^{h}\left(\mathbf{Z}\left(\zeta_{3}\right) \mathbf{Z} / 2 \times \mathbf{Z} / 2\right)$ surjects onto $L_{2}^{p}\left(\mathbf{Z}\left(\zeta_{3}\right) \mathbf{Z} / 2 \times \mathbf{Z} / 2\right)$ with kernel $\mathbf{Z} / 2$.

Proof. To begin note that

$$
L_{k}^{p}\left(\mathbf{Z}\left(\zeta_{3}\right) \mathbf{Z} / 2 \times \mathbf{Z} / 2\right)= \begin{cases}\mathbf{Z}^{4} & k=0 \\ (\mathbf{Z} / 2)^{3} & k=1 \\ \mathbf{Z}^{4} & k=2 \\ 0 & k=3 .\end{cases}
$$

Specifically, the generators for $L_{0}^{p}\left(\mathbf{Z}\left(\zeta_{3}\right) \mathbf{Z} / 2 \times \mathbf{Z} / 2\right)$ are $\langle 1\rangle$, and three forms given by specifying indexes at the four irreducible representations as

$$
\{0,2,0,0\},\{0,0,2,0\} \text {, and }\{0,0,0,2\}
$$

respectively. Thus, since $\left(1+2 \zeta_{3}^{2}\right) /\left(1+2 \zeta_{3}\right)=-1$ we see from (6.2) that each of the three classes above maps to the non-zero element in $\widehat{H}_{0}\left(\mathbf{Z} / 2 ; \widetilde{K}_{0}\left(\mathbf{Z}\left(\zeta_{3}\right) \mathbf{Z} / 2 \times \mathbf{Z} / 2\right)\right)$ in the first Ranicki-Rothenberg sequence in $(2.1)$. 
Similarly, the generators for $L_{1}^{p}\left(\mathbf{Z}\left(\zeta_{3}\right) \mathbf{Z} / 2 \times \mathbf{Z} / 2\right)$ are obtained using formations $\left(H \oplus H^{*}, K\right)$ which satisfy the property that projecting the second kernel $K$ to $H^{*}$ by factoring out $H$, gives a quotient of the form $H^{*} /\left(p_{H}(K)\right)=\mathbf{Z} / 3^{\varepsilon, \tau}$. Here $\varepsilon, \tau$ are each \pm 1 and represent the action of $\mathbf{Z}(\mathbf{Z} / 2 \times \mathbf{Z} / 2)$ on $\mathbf{Z} / 3$ via one of the 3 non-trivial irreducible representations. (7.2) follows.

We now calculate $\widetilde{K}_{0}\left(\mathbf{Z}\left(\zeta_{3}\right) D_{2^{i}},{ }^{-}\right)$.

LEMMA 7.6. $\widetilde{K}_{0}\left(\mathbf{Z}\left(\zeta_{3}\right) D_{8}\right)=(\mathbf{Z} / 2)^{2}$ with generators

$$
\left\{1,1,1,1+2 \zeta_{3}, 1\right\},\left\{1,1,1,1,1+2 \zeta_{3}\right\},
$$

where the first four places are the corresponding representations for the quotient group $\pi^{\mathrm{ab}}=\mathrm{Z} / 2 \times \mathrm{Z} / 2$ and the last place corresponds to the representation

$$
M: x \rightarrow\left(\begin{array}{cc}
0 & 1 \\
-1 & 0
\end{array}\right), \quad y \rightarrow\left(\begin{array}{cc}
1 & 0 \\
0 & -1
\end{array}\right) .
$$

(The proof is not much different from the proof of 7.4. Again from [W4]

$$
K_{1}\left(\widehat{\mathbf{Z}}_{2}\left(\zeta_{3}\right) D_{8}\right)=\mathbf{Z} / 6 \times(\mathbf{Z} / 2 \times \mathbf{Z} / 2) \times\left(\widehat{\mathbf{Z}}_{2}^{+}\right)^{10} .
$$

Hence, besides the generators listed for $K_{1}\left(\widehat{\mathbf{Z}}_{2}\left(\zeta_{3}\right) \mathbf{Z} / 2 \times \mathbf{Z} / 2\right)$ we require 2 more to give $K_{1}\left(\widehat{\mathbf{Z}}_{2}\left(\zeta_{3}\right) D_{8}\right)$, and these are

$$
1+\zeta_{3}(y x+1)\left(x^{2}-1\right), 1+\zeta_{3}\left(x^{2}-1\right) \text {. }
$$

The first of these has image $\left\{1,1,1,1,1+4 \zeta_{3}\right\}$ while the second has image $\left\{1,1,1,1,\left(1+2 \zeta_{3}\right)^{2}\right\}$. \{Note that the square appears in the last term because the reduced norm here is really just the ordinary determinant\}.)

COROLLARY 7.7. $L_{2}^{h}\left(\mathbf{Z}\left(\zeta_{3}\right) D_{8}\right)=(\mathbf{Z} / 2)^{2} \oplus \mathbf{Z}^{5}$, with one of the $\mathbf{Z} / 2$ 's surjecting onto the $\mathbf{Z} / 2$ in $L_{2}^{h}\left(\mathbf{Z}\left(\zeta_{3}\right) \mathbf{Z} / 2 \times \mathbf{Z} / 2\right)$.

There are two non-conjugate embeddings

$$
i_{\varepsilon}: \mathbf{Z} / 2 \times \mathbf{Z} / 2 \longrightarrow D_{8}, \quad \varepsilon=1,2,
$$

where $i_{1}(a)=x^{2}, i_{1}(b)=y$, and $i_{2}(a)=x^{2}, i_{2}(b)=x y$. They give induction maps

$$
i_{\varepsilon}^{!}: L_{k}^{h}\left(\mathbf{Z}\left(\zeta_{3}\right) \mathbf{Z} / 2 \times \mathbf{Z} / 2\right) \longrightarrow L_{k}^{h}\left(\mathbf{Z}\left(\zeta_{3}\right) D_{8}\right)
$$


and we have

LEMMA 7.9. When $k=2$ the induction maps $i_{\varepsilon}^{!}$are both nontrivial on the torsion class, mapping it onto the kernel of projection to $D_{8}^{\mathrm{ab}}=\mathbf{Z} / 2 \times \mathbf{Z} / 2$.

Proof. It is direct to see that the two maps $i_{1}$ and $i_{2}$ are conjugate via an outer automorphism of $D_{8}$. Thus it suffices to verify the result for $i_{1}$. For this inclusion we have

$$
i_{1}(\langle--\rangle)=M \quad(\text { as described in 7.6). }
$$

Thus, the unit $\left\{1,1,1,1+2 \zeta_{3}\right\}$ has image $\{1,1,1,1, v\}$ where $v$ is the determinant of the matrix $N=i_{1}^{!}\left(1-\left(\zeta_{3} / 2\right)(1-a-b+a b)\right)$, but calculating explicitly

$$
N=\left(\begin{array}{cc}
1 & 0 \\
0 & 1+2 \zeta_{3}
\end{array}\right)
$$

and from this the result follows.

More generally, for the dihedral group $D_{2^{i+1}}$ the two inclusions

$$
i_{\varepsilon}: \mathbf{Z} / 2 \times \mathbf{Z} / 2 \longrightarrow D_{2^{i+1}}, i_{\varepsilon}(a)=x^{2^{l-1}}, i_{1}(b)=y, i_{2}(b)=x y
$$

satisfy

$$
i_{\varepsilon}^{!}(\langle--\rangle)=M_{i} \quad \text { where } M_{i}(x)=\left(\begin{array}{cc}
0 & -1 \\
1 & \lambda_{i}
\end{array}\right), M_{i}(y)=\left(\begin{array}{ll}
0 & 1 \\
1 & 0
\end{array}\right)
$$

and $M_{i}$ is the unique irreducible faithful representation of $D_{2^{i+1}}$. In particular we have

THEOREM 7.10. $L_{2}^{h}\left(\mathbf{Z}\left(\zeta_{3}\right) D_{2^{i+1}}\right)=\mathbf{Z}^{i+3} \oplus(\mathbf{Z} / 2)^{i}$, and the map

$$
p_{i}: D_{2^{i+1}} \rightarrow D_{2^{i}} \quad\left(p_{i}(x)=x, p_{i}(y)=y\right)
$$

induces a surjection

$$
p_{i}^{!}: L_{2}^{h}\left(\mathbf{Z}\left(\zeta_{3}\right) D_{2^{i+1}}\right) \longrightarrow L_{2}^{h}\left(\mathbf{Z}\left(\zeta_{3}\right) D_{2^{i}}\right) .
$$

Moreover the torsion kernel of $p_{i}^{!}$is in the image of the induction maps

$$
i_{\varepsilon}^{!}: L_{2}^{h}\left(\mathbf{Z}\left(\zeta_{3}\right) \mathbf{Z} / 2 \times \mathbf{Z} / 2\right) \longrightarrow L_{2}^{h}\left(\mathbf{Z}\left(\zeta_{3}\right) D_{2^{l+1}}\right) \text {. }
$$

(The proof is direct and modeled on the proof of 7.9.)

Finally we consider the map

$$
\Omega_{4 k+2}\left(B_{\mathbf{Z} / 2 \times \mathbf{Z} / 2)}\right) L_{2}^{h}\left(\mathbf{Z}\left(\zeta_{3}\right) \mathbf{Z} / 2 \times \mathbf{Z} / 2\right)
$$

We do not attempt to calculate the entire map, but just enough for our purposes. Indeed the following result is sufficient. 
THEOREM 7.11. Let $f: S^{1} \times S^{1} \longrightarrow B_{\mathrm{Z} / 2 \times \mathbf{Z} / 2}$ send $e \otimes e$ to $e_{1} \otimes e_{1}$ where $e_{1}$ is the non-zero element in $H_{1}\left(B_{\mathbf{Z} / 2} ; \mathbf{Z}\right)=\mathbf{Z} / 2$, then

$$
\left\{S^{1} \times S^{1}, f\right\} \in \Omega_{2}\left(B_{\mathbf{Z} / 2 \times \mathbf{Z} / 2}\right)
$$

maps onto the non-trivial torsion class in $L_{2}^{h}\left(\mathbf{Z}\left(\zeta_{3}\right) \mathbf{Z} / 2 \times \mathbf{Z} / 2\right)$.

Proof. In [M-R] it is shown that the surgery problem

$$
S^{1} \times S^{1} \times K^{4 i+2} \stackrel{\mathrm{id} \times \mathrm{id} \times \sigma}{\longrightarrow} S^{1} \times S^{1} \times S^{4 i+2}
$$

is non-trivial when $S^{1} \times S^{1}$ is regarded as having fundamental group surjecting onto $\mathbf{Z} / 4 \times \mathbf{Z} / 2$. But $f$ factors as the composite

$$
S^{1} \times S^{1} \stackrel{g}{\longrightarrow} B_{\mathbf{Z} / 4 \times \mathbf{Z} / 2} \stackrel{h}{\longrightarrow} B_{\mathbf{Z} / 2 \times \mathbf{Z} / 2}
$$

where $g$ is induced from the surjection of fundamental groups, as is $h$.

$$
L_{2}^{h}\left(\mathbf{Z}\left(\zeta_{3}\right) \mathbf{Z} / 4 \times \mathbf{Z} / 2\right)=\mathbf{Z}^{6} \oplus \mathbf{Z} / 2
$$

and by default, the class of $\left\{S^{1} \times S^{1}, g\right\}$ in $L_{2}^{h}\left(\mathbf{Z}\left(\zeta_{3}\right) Z / 4 \times \mathbf{Z} / 2\right)$ is the non-trivial torsion element. But the map

$$
L_{2}^{h}\left(\mathbf{Z}\left(\zeta_{3}\right) \mathbf{Z} / 4 \times \mathbf{Z} / 2\right) \stackrel{h^{!}}{\longrightarrow} L_{2}^{h}\left(\mathbf{Z}\left(\zeta_{3}\right) \mathbf{Z} / 2 \times \mathbf{Z} / 2\right)
$$

is clearly surjective as well as injective on torsion. 7.11 follows.

8. The map $\Omega_{4 k}\left(B_{\pi}\right) \rightarrow L_{0}^{h}\left(Z\left(\zeta_{3}\right) \pi\right)$. In this section, when discussing bordism and homology we ignore odd torsion.

The classes in the torsion subgroup of $L_{0}^{h}\left(\mathbf{Z}\left(\zeta_{3}\right) \pi\right)$ are all induced from quaternion

$$
\left(\mathscr{Q}_{2^{1}}=\left\{x, y \mid x^{2^{1-2}}=y^{2}=(x y)^{2}\right\}\right)
$$

subquotients as we have seen in $\S 6$ (see also $\S 2$ of Part II, [M4]). Consequently, in order to show that the image of $\Omega_{*}\left(B_{\pi}\right)$ in $L_{0}^{h}\left(\mathbf{Z}\left(\zeta_{3}\right) \pi\right)$ is just the $\mathbf{Z}$ generated by $\langle 1\rangle$ it suffices to show that the same is true for the quaternion groups $\mathscr{Q}_{2^{\prime}}$. That is, we must show that

$$
\operatorname{im}\left(\Omega_{4 i}\left(B_{\mathscr{Q}_{2^{l}}}\right) \longrightarrow L_{0}^{h}\left(\mathbf{Z}\left(\zeta_{3}\right) \mathscr{Q}_{2^{l}},{ }^{-}\right)=\mathbf{Z}^{2^{l-2}+3} \oplus \mathbf{Z} / 2\right)
$$

is just the single copy of $\mathbf{Z}$ coming from the simply connected case: As in $\S 7$, we do this by an explicit analysis of individual elements in $\Omega_{4 *}\left(B_{Q_{2^{l}}}\right)$. However, here there is an important difference from the situation in $\S 7$ : we want a negative result, so, rather than look at a single element, we must check all elements. But generators for 
$\Omega_{4 *}\left(B_{\mathscr{Q}_{2^{\prime}}}\right)=\amalg_{r+s=4 *} H_{r}\left(B_{\mathscr{Q}_{2^{\prime}}} ; \Omega_{s}(p t)\right)$ are very hard to describe. Consequently, we use a technique which has been very helpful before, [M2], manifolds with $\mathbf{Z} / 2$-coefficients, and corresponding to them, $L$-groups with $\mathbf{Z} / 2$-coefficients.

The groups $L_{*}(A ; \mathbf{Z} / 2)$ are defined in terms of quadratic complexes $\mathscr{C}$ of dimension $n$ with boundary $\partial \mathscr{C}$ together with an equivalence $\partial \mathscr{C}=\delta \mathscr{C} \oplus \delta \mathscr{C}$, where $\delta \mathscr{C}$ is an $n-1$ dimensional Poincaré duality complex. These groups are easily calculated since there is an exact sequence

$$
\cdots \longrightarrow L_{k}^{h}(A) \stackrel{\times 2}{\longrightarrow} L_{k}^{h}(A) \longrightarrow L_{k}^{h}(A ; \mathbf{Z} / 2) \stackrel{\partial}{\longrightarrow} L_{k-1}^{h}(A) \stackrel{\times 2}{\longrightarrow} \cdots
$$

From (8.1) we have immediately

LEMMA 8.2.

$$
L_{k}\left(\mathbf{Z}\left(\zeta_{3}\right) ; \mathbf{Z} / 2\right)= \begin{cases}\mathbf{Z} / 2 & k=0 \\ 0 & k=1 \\ \mathbf{Z} / 2 & k=2 \\ 0 & k=3\end{cases}
$$

There is an analogous definition for $\Omega_{n}(X ; \mathbf{Z} / 2)$ in terms of $\mathbf{Z} / 2$ manifolds (manifolds $M^{n}$ with boundary $\partial M=\delta M \sqcup \delta M$ two isomorphic disjoint copies of a closed manifold $\delta M$ called the (geometric) Bochstein of $M$, and maps $f: M \rightarrow X$ which agree when restricted to the two pieces of $\partial M$ ). An equivalent way of thinking about Z/2-manifolds, is as possibly non-oriented manifolds with a chosen integral lifting of the first Stiefel-Whitney class, $w_{1}(M)$. The product of two $\mathbf{Z} / 2$-manifolds is again a $\mathbf{Z} / 2$-manifold, since $w_{1}(M \times N)$ is $w_{1}(M) \otimes 1+1 \otimes w_{1}(N)$, and this has an obvious integral lifting.

LeMMA 8.3. Let $M, N$ be $\mathrm{Z} / 2$-manifolds, then in the relevant $\mathbf{Z} / 2$ bordism group we have that

$$
\delta(M \times N)=(\delta M) \times N+M \times \delta N .
$$

Proof. $\delta(M \times N)=M \times \delta N \cup_{\partial M \times \delta N}(\delta M) \times N$, [M2]. But

$$
\partial M \times \delta N=\delta M \times \delta N \sqcup \delta M \times \delta N .
$$

In $\delta(M \times N)$ each copy of $\delta M \times \delta N$ has a trivial normal bundle, so a neighborhood looks like $I \times \delta M \times \delta N$. We can construct a $\mathbf{Z} / 2$ bordism of $\delta(M \times N)$ by simply attaching $I^{2} \times \delta M \times \delta N$ via the top 
and bottom copies of $I \times \delta M \times \delta N$, and the result of the bordism is the $\mathrm{Z} / 2$-manifold of 8.3 .

It is easy to compute $\mathbf{Z} / 2$-bordism since we have an exact sequence

$$
\cdots \Omega_{n}(X) \stackrel{\times 2}{\longrightarrow} \Omega_{n}(X) \longrightarrow \Omega_{n}(X ; \mathbf{Z} / 2) \stackrel{\partial}{\longrightarrow} \Omega_{n-1}(X) \stackrel{\times 2}{\longrightarrow} \cdots .
$$

In particular

$$
\begin{aligned}
\Omega_{*}(X ; \mathbf{Z} / 2) & =\coprod H_{r}\left(X ; \mathbf{\Omega}_{s}(p t ; \mathbf{Z} / 2)\right) \\
& =\coprod H_{r}(X ; \mathbf{Z} / 2) \otimes \Omega_{s}(p t, \mathbf{Z} / 2),
\end{aligned}
$$

and, using the product structure, generators are much easier to handle. When $A=\mathbf{Z}\left(\zeta_{3}\right) \pi$ and $X=B_{\pi}$ the composite maps

$$
\Omega_{n}\left(B_{\pi} ; \mathbf{Z} / 2\right) \longrightarrow L_{n}^{h}\left(\mathbf{Z}\left(\zeta_{3}\right) \pi ; \mathbf{Z} / 2\right)
$$

fit together with the composite maps

$$
\Omega_{n}\left(B_{\pi}\right) \longrightarrow L_{n}^{h}\left(\mathbf{Z}\left(\zeta_{3}\right) \pi\right)
$$

to give a map of exact sequences. Moreover, the pairings

$$
\Omega_{n}(p t ; \mathbf{Z} / 2) \otimes \Omega_{m}(X ; \mathbf{Z} / 2) \longrightarrow \Omega_{n+m}(X ; \mathbf{Z} / 2)
$$

and

$$
L^{n}(\mathbf{Z} ; \mathbf{Z} / 2) \otimes L_{m}^{h}\left(\mathbf{Z}\left(\zeta_{3}\right) \pi ; \mathbf{Z} / 2\right) \longrightarrow L_{n+m}^{h}\left(\mathbf{Z}\left(\zeta_{3}\right) \pi ; \mathbf{Z} / 2\right)
$$

also fit together in the obvious way to give commutative diagrams.

Of course, our main interest here is in what happens with the quaternion groups under the map

$$
e_{\zeta_{3}}: \Omega_{4 i}\left(B_{\mathscr{Q}_{2^{l}}}\right) \longrightarrow L_{0}^{h}\left(\mathbf{Z}\left(\zeta_{3}\right) \mathscr{Q}_{2^{\prime}}\right)
$$

Recall the well known result [Mu]

LEMMA 8.6.

$$
H_{4 i+j}\left(B_{\mathscr{Q}_{2^{l}}} ; \mathbf{Z} / 2\right)= \begin{cases}(\mathbf{Z} / 2)^{2} & j=1 \\ (\mathbf{Z} / 2)^{2} & j=2 \\ \mathbf{Z} / 2 & j=3 \\ \mathbf{Z} / 2 & j=0\end{cases}
$$

moreover $\mathrm{Sq}^{1}: H^{4 j+1} \rightarrow H^{4 j+2}$ is an isomorphism, while $H_{3}\left(B_{\mathscr{Q}_{2^{l}}} ; \mathbf{Z}\right)$ $=\mathbf{Z} / 2^{i}$, and $H_{4}\left(B_{\mathscr{Q}_{2^{i}}} ; \mathbf{Z}\right)=0$.

From (8.5), 8.6 we have

$$
\Omega_{*}\left(B_{\mathscr{Q}_{2^{l}}} ; \mathbf{Z} / 2\right)=\sum_{r+s=*} \Omega_{r}(p t ; \mathbf{Z} / 2) \otimes H_{S}\left(B_{\mathscr{Q}_{2^{l}}} ; \mathbf{Z} / 2\right)
$$


and we can choose representatives $V_{*}$ representing the elements in $H_{*}\left(B_{\mathscr{Q}_{2}} ; \mathrm{Z} / 2\right)$ so that $\delta V_{4 i+2}=V_{4 i+1}, \delta V_{4 i+2}^{\prime}=V_{4 i+1}^{\prime}$, while $\delta V_{4 i}=$ $2^{i-1} V_{4 i-1}$.

COROLLARY 8.7. $e_{\zeta_{3}}$ restricted to the torsion part of $\Omega_{4 i}\left(B_{\mathscr{Q}_{2^{l}}}\right)$ is zero.

Proof. Consider an element of the form $W^{n} \times V_{i}$. If we factor through the composite

$$
\begin{aligned}
& \Omega_{n}(p t ; \mathbf{Z} / 2) \times \Omega_{i}\left(B_{\mathscr{Q}_{2^{i}}} ; \mathbf{Z} / 2\right) \\
& \quad \longrightarrow L_{n}\left(\mathbf{Z}\left(\zeta_{3}\right) ; \mathbf{Z} / 2\right) \otimes L^{i}\left(\mathbf{Z} \mathscr{Q}_{2^{i}} ; \mathbf{Z} / 2\right) \longrightarrow L_{n+i}\left(\mathbf{Z}\left(\zeta_{3}\right) \mathscr{Q}_{2^{i}} ; \mathbf{Z} / 2\right)
\end{aligned}
$$

we see from 8.2 that $n$ must be even. Hence, since we are only interested in dimensions of the form $4 j, i$ must also be even. Since the generator in dimension 0 is in the image of $L_{i}\left(Z\left(\zeta_{3}\right)\right)$ where it is detected by the index, we can choose a basis for $\Omega_{4 i}(p t)$ so that $\mathbf{C} P^{2 i}$ is one of the generators and the others go to zero in $L_{4 i}\left(\mathbf{Z}\left(\zeta_{3}\right),{ }^{-}\right)$. But $M^{4 n} \times V^{4 i}$ has Bochstein $2^{i-1} M^{4 n} \times V^{4 i-1}$ if $M$ is oriented, and if $M$ has infinite order in $\Omega_{4 n}(p t)$ the Bochstein is non-zero, which implies there is no class in $\Omega_{4 i}\left(B_{\mathscr{Q}_{2^{2}}} ; \mathbf{Z} / 2\right)$ which is in the image of $\Omega_{4 i}\left(B_{\mathscr{Q}_{2^{i}}}\right)$ and goes non-trivially into $L_{0}^{h}\left(\mathbf{Z}\left(\zeta_{3}\right) \mathscr{Q}_{2^{\prime}} ; \mathbf{Z} / 2\right)$. A similar argument works for $M \times V_{4 i+2}, M \times V_{4 i+2}^{\prime}$, but it is even easier, since, by 8.3 , the Bochstein is already detected and non-trivial in $\mathbf{Z} / 2$ bordism. 8.7 follows.

Of course, 8.7 is the final step in the discussion of the map $\tau_{K}$, and the proofs of our main results are complete.

\section{REFERENCES}

[C-M] G. Carlsson and R. J. Milgram, The structure of odd L-groups, Proc. 1978 Waterloo Conference on Algebraic Topology, Springer-Verlag Lecture notes, 741 (1979), 1-72.

[C-S] S. Cappell and J. Shaneson, A counterexample on the oozing problem for closed manifolds, Proceedings 1978 Aarhus Conference on Algebraic Topology, Springer-Verlag Lecture notes, 763 (1979), 627-634.

[Cl] $\quad F$. Clauwens, The $K$-theory of almost symmetric forms, in Topological structures II, 1979, Mathematical Centre Tracts, Proceedings of the symposium in Amsterdam, October 31-November 2, 1978, 115 (1979), 41-49. 
$[\mathrm{F}]$

$[\mathrm{H}]$

[H-M]

[H-M-T-W] I. Hambleton, R. J. Milgram, L. Taylor, and B. Williams, Surgery on manifolds with finite fundamental group, Proc. Lond. Math. Soc., (3) 56 (1988), 349-379.

$[\mathrm{K}]$

J. M. Fontaine, Sur la décomposition des algèbres de groupes, Ann. Sci. École Norm. Sup., 4 (1971), 121-180.

I. Hambleton, Projective surgery obstructions on closed manifolds, Algebraic $K$-theory, Oberwolfach 1980, Part II, Lecture Notes in Mathematics, 967 (Springer, Berlin, 1982), 101-131.

I. Hambleton and R. J. Milgram, The surgery obstruction groups for finite 2-groups, Invent. Math., 63 (1980), 33-52.

M. Kolster, Computations of Witt groups of finite groups, Math. Ann., 241 (1979), 129-158.

W. Lück and A. Ranicki, to appear.

R. J. Milgram, Solution of the Schur subgroup problem, mimeo. Stanford, 1978.

—, Surgery with coefficients, Ann. of Math., 2 (100) (1974), 194-248. Odd index units in cyclotomic number fields and applications, Algebraic $K$-theory, Evanston 1980, Springer-Verlag Lecture Notes in Math., 854 (1980), 269-298.

_, Surgery with finite fundamental group II: the oozing conjecture, Pacific J. Math., 151 (1991), 117-150.

R. J. Milgram and R. Oliver, $P$-adic logarithms for transcendence degree 1 extensions and applications, to appear.

R. J. Milgram and A. Ranicki, Some product formulae for nonsimply connected surgery problems, Trans Amer. Math. Soc., 297 (1986), 383413.

J. Milnor and D. Husemoller, Symmetric Bilinear Forms, SpringerVerlag (1972).

J. Morgan and D. Sullivan, The transversality characteristic class and linking cycles in surgery theory, Ann. of Math., 99 (1974), 463-544.

H. J. Munkholm, Mod 2 cohomology of $D_{2^{n}}$ and extensions by 2, Conference on Algebraic Topology, Univ. of Illinois at Chicago Circle (1968) 234-252 .

R. Oliver, $S K_{1}$ for finite group rings II, Math. Scand., 47 (1980), 195231.

W. Pardon, The exact sequence of a localization for Witt groups II: $n u-$ merical invariants of odd dimensional surgery obstructions, Pacific J. Math., 102 (1982), 123-170.

A. Ranicki, On the algebraic L-theory of semisimple rings, J. Algebra, 50 (1978), 242-243.

(40) (1980), 87-283.

L. Taylor-B. Williams, Surgery on closed manifolds, mimeo. Notre Dame University (1980).

C. T. C. Wall, Surgery on Compact Manifolds, Academic Press (1970)Formulae for surgery obstructions, Topology, 15 (1976), 189-210.

_- On the classification of Hermitian forms VI: group rings, Ann. of Math., 108 (1976), 1-80.

, Norms of units in group rings, Proc. London Math. Soc., 29 (1974), 593-632. 
[WA] L. Washington, Introduction to Cyclotomic Fields, Springer Graduate Texts in Mathematics, 83 (1982).

Received March 23, 1988 and, in revised form, May 25, 1989. Research supported in part by grants from the NSF.

University of California, SAN Diego

LA Jolla, CA 92093

AND

STANFORD UNIVERSITY

STANFORD, CA 94305-2125 



\section{PACIFIC JOURNAL OF MATHEMATICS EDITORS}

\author{
V. S. VARADARAJAN \\ (Managing Editor) \\ University of California \\ Los Angeles, CA 90024-1555-05 \\ Herbert Clemens \\ University of Utah \\ Salt Lake City, UT 84112 \\ THOMAS ENRIGHT \\ University of California, San Diego \\ La Jolla, CA 92093
}

Nicholas ERCOLANI

University of Arizona

Tucson, AZ 85721

R. FINN

Stanford University

Stanford, CA 94305

VAUGHAN F. R. JONES

University of California

Berkeley, CA 94720

STEVEN KeRCKHOFF

Stanford University

Stanford, CA 94305
C. C. MOORE

University of California

Berkeley, CA 94720

Martin ScharlemanN

University of California

Santa Barbara, CA 93106

HAROLd STARK

University of California, San Diego

La Jolla, CA 92093

\section{ASSOCIATE EDITORS}
R. ARENS
E. F. BECKENBACH
B. H. NeUMANN
F. WolF
K. Yoshida (1906-1982)
(1904-1989)

SUPPORTING INSTITUTIONS

UNIVERSITY OF ARIZONA

UNIVERSITY OF BRITISH COLUMBIA

CALIFORNIA INSTITUTE OF TECHNOLOGY

UNIVERSITY OF CALIFORNIA

MONTANA STATE UNIVERSITY

UNIVERSITY OF NEVADA, RENO

NEW MEXICO STATE UNIVERSITY

OREGON STATE UNIVERSITY
UNIVERSITY OF OREGON

UNIVERSITY OF SOUTHERN CALIFORNIA

STANFORD UNIVERSITY

UNIVERSITY OF HAWAII

UNIVERSITY OF TOKYO

UNIVERSITY OF UTAH

WASHINGTON STATE UNIVERSITY

UNIVERSITY OF WASHINGTON 


\section{Pacific Journal of Mathematics}

\section{Vol. 151, No. $1 \quad$ November, 1991}

Fernanda Maria Botelho, Rotational entropy for annulus endomorphisms . . . 1

David F. Cowan, Some infinite chains in the lattice of varieties of inverse

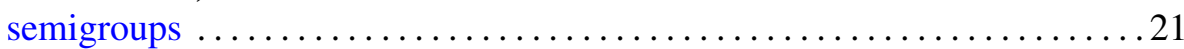

S. Greco and G. Raciti, The Lüroth semigroup of plane algebraic curves . . . 44

Kang-Tae Kim, Automorphism groups of certain domains in $\mathbf{C}^{n}$ with a

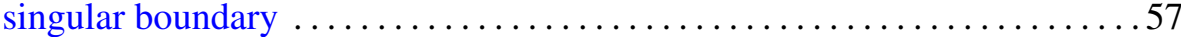

R. James Milgram, Surgery with finite fundamental group. I: The

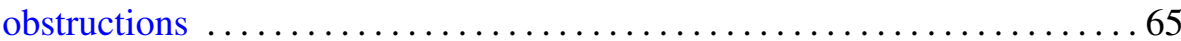

R. James Milgram, Surgery with finite fundamental group. II: The oozing

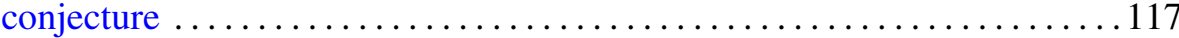

Shahriar Shahriari, On central type factor groups $\ldots \ldots \ldots \ldots \ldots \ldots \ldots 151$

Lynne Walling, Hecke eigenforms and representation numbers of quadratic forms ............................................... 179 